\title{
Linhas de curvatura direcional média em superfícies imersas em $R^{4}$
}

Luis Fernando de Osório Mello

TESE APRESENTADA AO

INSTITUTO DE MATEMÁTICA E ESTATÍSTICA

DA UNIVERSIDADE DE SÃO PAULO

PARA OBTENÇÃO DO GRAU DE

DOUTOR EM MATEMÁTICA APLICADA

Área de Concentração: Equações Diferenciais e Sistemas Dinâmicos

Orientador: Prof. Dr. Jorge Manuel Sotomayor Tello

São Paulo - Agosto de 2001

Durante a elaboração deste trabalho o autor recebeu auxílio financeiro da CAPES, convênio PICDT. 


\title{
Linhas de curvatura direcional média \\ em \\ superfícies imersas em $R^{4}$
}

\author{
Este exemplar corresponde à redação final \\ da tese de doutorado devidamente \\ corrigida e defendida por \\ Luis Fernando de Osório Mello \\ e aprovada pela comissão julgadora.
}

São Paulo, agosto de 2001.

Banca examinadora:

- Prof. Dr. Jorge Manuel Sotomayor Tello (Orientador) - IME-USP

- Prof. Dr. Paolo Piccione - IME-USP

- Prof. Dr. Carlos Teobaldo Gutiérrez Vidalón - ICMSC-USP

- Prof. Dr. Maria Aparecida Soares Ruas - ICMSC-USP

- Prof. Dr. Ronaldo Alves Garcia - IME-UFG 
À Edinita, companheira nesta jornada, e aos meus filhos

Larissa e Luis Gustavo. 


\section{Agradecimentos}

Muitas são as pessoas com as quais eu gostaria de compartilhar este momento, numa retribuição ao apoio que me deram durante a elaboração deste trabalho, seja no aspecto profissional, seja no aspecto pessoal. Sem a ajuda de vocês, sinceramente, eu não conseguiria. Torno explícita minha dívida, e agradeço a todos vocês de coração.

Aos professores Jorge Sotomayor, pela orientação deste trabalho e pela convivência saudável durante estes quatro anos, e Ronaldo Garcia, sempre disponível e atencioso.

Aos colegas e amigos Roberto Barreto, Ana Lúcia e Marcelo Messias, nobres companheiros, com quem dividi angústias e esperanças.

Aos professores do ICI-EFEI, que possibilitaram meu afastamento, e em especial ao meu amigo e compadre Baêta, pelo constante incentivo.

À minha mãe Dicéa, aos meus irmãos Rogério e Márcia, e à Dona Ernestina, pessoas que muito estimo e que sempre me incentivaram.

Aos meus amigos José Antônio, José Aloísio de Oliveira e Joccitiel, ao meu cunhado Marcos e à Tia Maria Antônia, pelo constante apoio.

Ao meu pai Hélcio e ao Seu Manoel, já falecidos mas sempre presentes em meu pensamento.

Luis Fernando de Osório Mello. 


\section{Resumo}

Para qualquer superfície imersa em $R^{4}$ podemos associar sua configuração direcional média, a qual está definida pelas famílias das curvas sobre a superfície cujo vetor curvatura normal está na direção do vetor curvatura média, aqui chamadas de linhas de curvatura direcional média, e suas singularidades, denominadas aqui de $H$-singularidades. Isto é um análogo natural ao conceito de configuração principal das superfícies imersas em $R^{3}$. Estudamos aqui as $H$-singularidades genéricas, análogas aos pontos umbílicos Darbouxianos, e a hiperbolicidade das linhas de curvatura direcional média periódicas, chamadas ciclos de curvatura direcional média. Condições suficientes em termos das $H$-singularidades, dos ciclos de curvatura direcional média e do comportamento assintótico das outras linhas de curvatura direcional média são fornecidas para garantir a estabilidade estrutural direcional média de superfícies imersas em $R^{4}$. 


\begin{abstract}
To any immersed surface into $R^{4}$ is associated its directional mean configuration, which consists on the family of the lines whose normal curvature vector points in the direction of the mean curvature vector, called normally mean directionally curved lines, and their singularities, called $\mathrm{H}$ singularities. This is a natural analog of the principal configuration on an immersed surface in $R^{3}$. We analyze here the concepts of the generic $H$-singularities, analogous to Darbouxian umbilic points, and the hyperbolicity of the closed normally directionally mean curved lines, called directional mean curvature cycles. Sufficient conditions in terms of the $\mathrm{H}$ singularities, directional mean curvature cycles and asymptotic behavior of all the other normally mean directionally curved lines are provided to guarantee the directional mean curvature structural stability of surfaces immersed in $R^{4}$.
\end{abstract}




\section{Sumário}

Capítulo 1. Introdução 1

Capítulo 2. Equação Diferencial Para as Linhas de Curvatura

Direcional Média

10

2.1. A Elipse de Curvatura 10

2.2. A Equação Diferencial das Linhas de Curvatura

Direcional Média 19

Capítulo 3. Estudo das $H$-singulariadades $\quad 24$

3.1. O Caso dos Pontos Mínimos 26

3.2. O Caso dos Pontos de Inflexão 29

Capítulo 4. $H$-singularidades do Tipo $S$

4.1. O Caso dos Pontos Mínimos 39

4.2. O Caso dos Pontos de Inflexão 44

Capítulo 5. Linhas de Curvatura Direcional Média Periódicas 46 
Capítulo 6. Um Caso Especial 55

6.1. A Projeção de uma Superfície Imersa em $R^{3}$ em $S^{3} \quad 55$

6.2. Estudo dos Campos de Direções 64

6.3. Quadro Comparativo das Imersões 68

6.4. Superfícies na Esfera $S^{3} \quad 68$

Capítulo 7. Estabilidade Estrutural Direcional Média 72

Capítulo 8. Topologia e Configuração Direcional Média 80

$\begin{array}{lr}\text { Capítulo 9. Conclusões } & 84\end{array}$

Capítulo 10. Referências Bibliográficas $\quad 86$ 


\section{Capítulo 1. Introdução}

Na Geometria Diferencial Clássica dedica-se especial atenção aos tópicos que tratam da curvatura de uma superfície imersa em $R^{3}$. Dentro destes destacamse os estudos envolvendo as curvaturas principais e as funções a elas associadas, como, por exemplo, as curvaturas Gaussiana e Média [Car]. Intimamente ligadas às curvaturas principais temos as direções principais e suas curvas integrais (as linhas de curvatura principal) e suas singularidades (os pontos umbílicos). Abordaremos inicialmente este assunto, pois ele nos conecta com a Teoria Qualitativa das Equações Diferenciais Ordinárias que é útil no contexto em que este trabalho se insere.

As linhas de curvatura principal de uma superfície imersa em $R^{3}$ são as curvas sobre a superfície tais que ao longo de suas direções tangentes, denominadas direções principais, estas se curvam extremalmente. Tais curvaturas extremais denominam-se curvaturas principais e seus valores são obtidos através da curvatura normal avaliada nas direções principais. As direções principais são, em geral, determinadas por duas retas ortogonais. Deste modo, fora dos pontos umbílicos, onde as curvaturas principais coincidem, as direções principais determinam dois campos de direções tangentes à superfície, denominados campos de direções principais. Os pontos umbílicos são considerados singularidades destes 
campos. A rede formada pelas curvas integrais dos campos de direções principais, juntamente com os pontos umbílicos definem o que chamamos de configuração principal da superfície; ela constitui um análogo natural ao retrato de fase para campos de vetores em superfícies.

Euler [Eul] foi o primeiro matemático a inserir os métodos do Cálculo Infinitesimal em Geometria e o responsável pelas definições básicas acerca das curvaturas principais, em 1760.

No entanto, foi Monge [Mon], em 1796, o primeiro a destacar a importância da configuração principal de uma superfície. A ele devemos a descrição da configuração principal não trivial do elipsóide de três eixos distintos, denominado elipsóide de Monge. Tal fato "coloca Monge como indiscutível precursor da Teoria Qualitativa das Equações Diferenciais, quase um século mais tarde fundada e sistematizada por Poincaré e Liapunov, e aprimorada por Andronov, Pontrjagin, Peixoto,...", conforme [Sot]. A continuação dos trabalhos de Monge deveu-se a seu discípulo Dupin, o qual obteve um método de descrição de numerosas configurações principais através do conhecido resultado acerca das famílias triplamente ortogonais de superfícies. Como um caso particular podemos citar as quádricas homofocais, onde se encaixa o elipsóide de Monge.

Um avanço significativo no estudo das configurações principais foi dado por Darboux [Dar], em 1896, que descreveu os pontos umbílicos genéricos, caracterizando-os em termos das condições algébricas nas terceiras derivadas da superfície. Em sua homenagem tais pontos umbílicos receberam o nome de Darbouxianos.

Por quase um século o estudo da configuração principal de uma superfície permaneceu em repouso, sem que resultados substanciais fossem obtidos. Vale ressaltar que no período mencionado houve o nascimento, com Poincaré, e a 
propagação da Teoria Qualitativa das Equações Diferenciais, a qual, no entanto, não se alastrou no ramo da Geometria Diferencial Clássica.

Somente em 1982, com os trabalhos de Gutierrez e Sotomayor, coletados em [GS1], é que o estudo das configurações principais voltou ao cenário e se fortaleceu, ganhou corpo e se amoldou às modernas técnicas da Teoria Qualitativa das Equações Diferenciais e dos Sistemas Dinâmicos, com a introdução de novos conceitos como Estabilidade Estrutural e Genericidade. Denominaremos esta sua nova face de Teoria Qualitativa das Equações Diferenciais da Geometria, com a permissão do professor Sotomayor que utiliza este nome em um de seus cursos na USP.

Em [GS1] Gutierrez e Sotomayor definiram a classe GS das imersões de classe $C^{r}$ de superfícies compactas em $R^{3}$ que satisfazem as seguintes condições, reminiscentes das de Peixoto para Equações Diferenciais Ordinárias [Pei]:

i) Todos os pontos umbílicos são Darbouxianos;

ii) Todos os ciclos principais são hiperbólicos;

iii) Não existe conexão ou autoconexão de separatrizes umbílicas;

iv) O conjunto limite de cada linha de curvatura principal está contido no conjunto dos pontos umbílicos e ciclos principais.

Estes autores demonstraram que toda imersão pertencente ao conjunto GS é estruturalmente estável, bem como mostraram que esta classe é aberta e densa no conjunto de todas as imersões de superfícies compactas em $R^{3}$, com topologias adequadas. Para um maior esclarecimento dos termos acima empregados, bem 
como outros aspectos a respeito das configurações principais, recomendamos o recente estudo [GS2], no qual encontra-se uma lista de referências atualizadas sobre $o$ assunto.

As noções de curvaturas e direções principais, linhas de curvatura principal, pontos umbílicos, redes e configurações principais, bem como outras, podem ser estendidas para as hipersuperfícies imersas em $R^{4}$. Partindo de tal situação Garcia em [Ga1], em 1989, estendeu parcialmente os resultados de Gutierrez e Sotomayor para as hipersuperfícies compactas imersas em $R^{4}$.

No que foi dito acima tudo se refere à codimensão 1. Relataremos a seguir o que sabemos a respeito dos campos de direções e suas singularidades para as imersões de superfícies em $R^{4}$. Para o estudo geral das superfícies em $R^{4}$ podemos citar os marcos de referência Forsyth [For], Wong [Won] e Little [Lit]. Este último apresenta alguns exemplos de campos de direções, com suas construções baseadas na elipse de curvatura, e suas singularidades. Relaciona aspectos locais, como por exemplo, o índice do campo de direções nas singularidades isoladas, com aspectos globais, utilizando para isto hipóteses adicionais como a compacidade da superfície. Encontra uma interessante relação entre o índice de um campo de direções (chamado aqui de campo de direções de curvatura direcional média) e o índice do campo de vetores curvatura média que compartilham da mesma singularidade, apresentando, no entanto, um equívoco com relação ao sinal envolvido nesta relação. Mais especificamente, este equívoco aparece na interpretação do sinal da curvatura normal (veja página 11). Este equívoco foi percebido e corrigido por Asperti em [Asp]. Neste trabalho Asperti refaz os principais resultados de [Lit]. Apresentamos neste nosso trabalho uma outra versão deste resultado de Little e Asperti. Vale observar que nas referências 
acima nada se encontra nos moldes dos resultados de Gutierrez-Sotomayor, ou seja, nos moldes da Teoria Qualitativa das Equações Diferenciais da Geometria.

Apenas recentemente foi dado ênfase ao estudo mais detalhado dos campos de direções e suas singularidades sobre as superfícies em $R^{4}$. Podemos citar o trabalho [GGST] de Gutierrez, Guadalupe, Sotomayor e Tribuzy, de 1987, onde estudaram as linhas de curvatura em superfícies mínimas imersas em $R^{4}$, e o trabalho de Gutierrez, Guadalupe, Tribuzy e Guíñez [GGTG], de 1997, onde estes autores fizeram um estudo detalhado a respeito das singularidades genéricas dos campos de direções cujas curvas integrais chamaram de linhas de curvatura. $\mathrm{O}$ motivo deste nome vem do fato de que na definição deste campo de direções utilizamos os pontos sobre a elipse de curvatura que determinam os maiores e menores eixos da mesma (veja página 10). Vale observar que o estudo destas singularidades foi feito em coordenadas especiais, no caso, coordenadas isotérmicas.

Deve-se a Garcia e Sotomayor em [GaS1] o estudo mais abrangente dos campos de direções acima e suas singularidades. Estes autores adotaram um nome mais sugestivo para as curvas integrais destes campos de direções, chamando-as de linhas de curvatura axial, bem como passaram a denominar suas singularidades de pontos axiumbílicos, pela estreita analogia com o caso de $R^{3}$. Convém salientar que o trabalho [GaS1] contém muito mais. Ele trata ainda do estudo dos ciclos e da estabilidade estrutural, nos moldes das imersões de superfícies em $R^{3}$, como acima mencionado.

Na mesma linha destes trabalhos encontramos ainda o estudo de Garcia, Mochida, Fuster e Ruas [GMFR] sobre as singularidades genéricas dos campos de direções assintóticas (veja página 15). Tais singularidades são denominadas pontos de inflexão. Aqui o apelo com o $R^{3}$ é, de certa forma, artificial, pois esta 
construção em nada tem a ver com as curvas assintóticas que aparecem em superfícies imersas em $R^{3}$. A única semelhança reside no fato de que estes campos estão definidos apenas em certas regiões das superfícies, denominadas regiões hiperbólicas, como no caso do $R^{3}$. No trabalho acima prova-se que genericamente o índice das singularidades destes campos é $\pm 1 / 2$, e como sobre as superfícies localmente convexas estes campos estão globalmente definidos, demonstra-se o seguinte teorema: qualquer esfera, genericamente mergulhada como uma superfícies localmente convexa em $R^{4}$, possui pelo menos 4 pontos de inflexão. Este tipo de resultado é típico. De posse dos índices das singularidades genéricas e da característica de Euler-Poincaré da superfície compacta chega-se a ele facilmente. No presente trabalho redemonstramos este resultado, utilizando os mesmos pontos de inflexão, mas agora olhando-os como singularidades de outro campo de direções.

Aqui vale uma digressão. Relacionada ao número de singularidades de um campo de direções sobre uma superfície compacta de $R^{3}$ temos a Conjectura de Carathéodory, a qual afirma que sobre todo ovalóide (superfície compacta com curvatura Gaussiana positiva) existem pelo menos dois pontos umbílicos. Esta conjectura permanece em aberto, pelo menos para o caso não analítico. Recentes contribuições ao entendimento da mesma têm sido dadas, como, por exemplo, o trabalho [SMe], onde referências para o assunto podem ser encontradas.

No estudo realizado por Garcia e Sotomayor em [GaS1], mencionado acima, aparece de forma natural uma equação diferencial quártica, a equação diferencial das linhas de curvatura axial. Ocorre que quando a imersão da superfície em $R^{4}$ está, em particular, contida em um hiperplano, esta equação quártica fatora-se num produto de duas equações diferenciais quadráticas, sendo uma delas a equação diferencial das linhas de curvatura principal e a outra 
denominada de equação diferencial das linhas de curvatura média. O estudo relacionado a esta última equação foi feito por Garcia e Sotomayor em [GaS2]. Neste trabalho os autores desenvolveram todo o estudo da estabilidade estrutural: fizeram o estudo das singularidades genéricas, dos ciclos hiperbólicos, do comportamento dos conjuntos limites das linhas de curvatura média e das separatrizes das singularidades genéricas, como no caso das linhas de curvatura principal supra citado.

Consideremos agora a imersão de uma superfície em $R^{3}$ e façamos a sua composição com a aplicação (inversa da) projeção estereográfica de $R^{3}$ em $S^{3}$. Temos assim, naturalmente, uma imersão de uma superfície em $R^{4}$. Em [Lit] Little mostrou que com esta construção as linhas de curvatura principal da imersão em $R^{3}$ são levadas nas linhas assintóticas da imersão em $R^{4}$, bem como os pontos umbílicos da imersão em $R^{3}$ são levados nos pontos de inflexão da imersão em $R^{4}$. Uma pergunta natural surge: Em quais linhas são aplicadas as linhas de curvatura média estudadas por Garcia e Sotomayor em [GaS2] ? Uma resposta para esta pergunta é dada no decorrer deste trabalho. De fato, fazemos algo um pouco mais abrangente. Desenvolvemos todo o estudo da estabilidade estrutural para um certo tipo de campo de direções, denominado neste trabalho de campo de direções de curvatura direcional média, cuja definição pode ser vista na página 17 , devido à sua ligação com o caso em $R^{3}$. As curvas integrais deste campo são chamadas de linhas de curvatura direcional média. Mais especificamente, este trabalho se desenvolve do seguinte modo.

No capítulo 2 fazemos uma revisão dos principais conceitos a respeito de uma imersão de uma superfície em $R^{4}$. Revisamos as formas fundamentais da imersão, a elipse de curvatura e as funções a ela associadas, como o vetor curvatura normal, o vetor curvatura média e a curvatura normal. Fazemos a 
construção do campo de direções de curvatura direcional média e determinamos a sua equação diferencial. Resulta que esta equação diferencial é uma equação diferencial binária, como as das linhas de curvatura principal para imersões em $R^{3}$.

No capítulo 3 damos destaque ao estudo das singularidades dos campos de direções de curvatura direcional média, chamadas aqui de $H$-singularidades. Mostramos que elas ocorrem nos pontos de inflexão ou nos pontos onde o vetor curvatura média $H$ é nulo, ou seja, nas singularidades do campo de vetores curvatura média. Exploramos neste capítulo o conceito de $H$-singularidade transversal, onde as curvas que definem a $H$-singularidade encontram-se transversalmente, e o conceito de índice (do campo de direções) na $H$ singularidade. Uma relação entre o índice da $H$-singularidade e o índice da singularidade do campo de vetores curvatura média é estabelecida.

No capítulo 4 estudamos uma classe das $H$-singularidades denominada de tipo $S_{i}, i=1,2,3$. O subíndice refere-se ao número de separatrizes da $H$ singularidade. Estas singularidades são análogas aos pontos umbílicos Darbouxianos das imersões em $R^{3}$. Fazemos tal estudo na carta de Monge.

O capítulo 5 é dedicado ao estudo das linhas de curvatura direcional média periódicas ou ciclos de curvatura direcional média. Determinamos a derivada da aplicação de primeiro retorno como uma expressão integral envolvendo invariantes geométricos da imersão ao longo do ciclo. Com isto temos uma caracterização dos ciclos hiperbólicos.

No capítulo 6 estudamos a situação onde a imersão de uma superfície em $R^{4}$ é oriunda de uma imersão de uma superfície em $R^{3}$, via (inversa da) projeção estereográfica de $R^{3}$ em $S^{3}$. Estabelecemos uma correspondência entre as formas fundamentais, entre alguns campos de direções e entre suas singularidades nos 
dois casos considerados. Obtemos ainda um resultado que garante a fatoração da equação diferencial quártica das linhas de curvatura axial num produto de duas equações diferenciais quadráticas, uma delas sendo a equação diferencial das linhas assintóticas e a outra a equação diferencial das linhas de curvatura direcional média.

No capítulo 7 definimos o conceito de configuração direcional média, de modo análogo ao de configuração principal para o caso em $R^{3}$. De posse dos resultados referentes às $H$-singularidades de tipo $S_{i}$ e aos ciclos de curvatura direcional média hiperbólicos desenvolvemos o estudo da estabilidade estrutural das configurações direcionais médias.

Tendo em mãos o resultado sobre a genericidade das $H$-singularidades de tipo $S_{i}$, visto no capítulo 7 , podemos obter algumas informações acerca de questões globais das superfícies, adicionando hipóteses, como por exemplo, a compacidade e conexidade das mesmas. Isto é feito no capítulo 8.

No capítulo 9 apresentamos conclusões a respeito deste trabalho e listamos alguns problemas em aberto dentro deste assunto.

No final apresentamos uma lista contendo as principais referências bibliográficas utilizadas neste trabalho. 


\section{Capítulo 2. Equação Diferencial para as Linhas de Curvatura Direcional Média}

Este capítulo está dividido em duas partes. $\mathrm{Na}$ primeira delas estabeleceremos as principais definições dos objetos tratados neste trabalho, visto que uma grande parte delas não é de uso cotidiano. Fixaremos ainda as notações empregadas ao longo do trabalho. $\mathrm{Na}$ segunda seção encontraremos a equação diferencial das linhas de curvatura direcional média, equação esta que nos acompanhará ao longo de todo o trabalho.

\subsection{A Elipse de Curvatura}

Seja $\alpha: M^{2} \rightarrow R^{4}$ uma imersão de classe $C^{r}, r \geq 3$, de uma superfície regular e orientada $M$ em $R^{4}$, o qual está orientado e munido do produto interno euclidiano $<,>$. Denotemos, respectivamente, por $T M$ e $N M$ os fibrados tangente e normal de $\alpha$ e por $T_{p} M$ e $N_{p} M$ as respectivas fibras, isto é, os planos tangente e normal em $p \in M$. Assumamos que $(u, v)$ seja uma carta positiva de $M$ e que $\left\{\alpha_{u}, \alpha_{v}, N_{1}, N_{2}\right\}$ seja um referencial positivo de $R^{4}$, onde $\left\{N_{1}, N_{2}\right\}$ é um 
referencial de campos de vetores ortonormais a $\alpha$. Nesta carta $(u, v)$, a primeira forma fundamental de $\alpha, I_{\alpha}$, é dada por

$$
I=I_{\alpha}=\langle d \alpha, d \alpha\rangle=E d u^{2}+2 F d u d v+G d v^{2},
$$

onde

$$
E=\left\langle\alpha_{u}, \alpha_{u}\right\rangle, F=\left\langle\alpha_{u}, \alpha_{v}\right\rangle \text { e } G=\left\langle\alpha_{1}, \alpha_{1}\right\rangle
$$

A segunda forma fundamental de $\alpha, I I_{\alpha}$, é definida por

$$
I I=I I_{\alpha}=\left\langle d^{2} \alpha, N_{1}\right\rangle N_{1}+\left\langle d^{2} \alpha, N_{2}\right\rangle N_{2}=I I_{1, \alpha} N_{1}+I I_{2, \alpha} N_{2},
$$

onde

$$
I_{i}=I I_{i, \alpha}=e_{i} d u^{2}+2 f_{i} d u d v+g_{i} d v^{2}
$$

e

$$
e_{i}=\left\langle\alpha_{u m}, N_{i}\right\rangle, f_{i}=\left\langle\alpha_{u v}, N_{i}\right\rangle \text { e } g_{i}=\left\langle\alpha_{v w}, N_{i}\right\rangle \text {, para } i=1,2
$$

Temos as seguintes funções associadas à imersão $\alpha$ (para maiores detalhes veja [Lit]):

1) o vetor curvatura média de $\alpha$

onde

$$
H=H_{\alpha}=H_{1} N_{1}+H_{2} N_{2} \text {, }
$$

$$
H_{i}=H_{i, \alpha}=\frac{E g_{i}-2 F f_{i}+G e_{i}}{2\left(E G-F^{2}\right)}, \text { para } i=1,2 ;
$$

2) a curvatura normal de $\alpha$

$$
k_{N}=k_{N, \alpha}=\frac{E\left(f_{1} g_{2}-f_{2} g_{1}\right)-F\left(e_{1} g_{2}-e_{2} g_{1}\right)+G\left(e_{1} f_{2}-e_{2} f_{1}\right)}{2\left(E G-F^{2}\right)} ;
$$

3) a resultante $\Delta$ de $I I_{1, \alpha}$ e $I I_{2, \alpha}$

$$
\Delta=\Delta_{\alpha}=\frac{1}{4\left(E G-F^{2}\right)} \operatorname{det}\left[\begin{array}{cccc}
e_{1} & 2 f_{1} & g_{1} & 0 \\
e_{2} & 2 f_{2} & g_{2} & 0 \\
0 & e_{1} & 2 f_{1} & g_{1} \\
0 & e_{2} & 2 f_{2} & g_{2}
\end{array}\right] \text {; }
$$


4) o vetor curvatura normal de $\alpha, \eta=\eta_{\alpha}$, é definido por $\eta: T M \rightarrow N M$, onde

$$
\eta(p, w)=\frac{I I(p, w)}{I(p, w)}
$$

De modo análogo ao estudo das imersões de superfícies em $R^{3}$, podemos olhar o vetor curvatura normal da seguinte maneira:

Para cada vetor unitário $w \in T_{p} M$, seja $\gamma:(-\varepsilon, \varepsilon) \rightarrow M$ uma curva, parametrizada pelo comprimento de arco $s$, tal que $\gamma(0)=p$ e $\gamma^{\prime}(0)=w$. Deste modo, $\eta(p)(w)=\eta(p, w)$ é a projeção de $\gamma^{\prime \prime}(0)$ sobre o plano normal $N_{p} M$. Esta interpretação independe da particular escolha da curva $\gamma$.

Com vistas na simplificação dos cálculos envolvidos, vamos adotar uma carta $(u, v): M \rightarrow R^{2}$ de modo que, nesta carta, a primeira forma fundamental esteja diagonalizada, com $E=G=1$ e $F=0$. Deste modo, $\left\{\alpha_{u}, \alpha_{v}\right\}$ é um referencial ortonormal de $T_{p} M$ e todo $w \in S^{1} \subset T_{p} M$ pode ser escrito como $w=\varphi(t)=\cos t \alpha_{u}+\operatorname{sen} t \alpha_{v}$.

Com estas considerações, temos

$$
\eta(p)(w)=\eta(p)(\varphi(t))=\left[\begin{array}{l}
e_{1} \cos ^{2} t+2 f_{1} \cos t \operatorname{sen} t+g_{1} \operatorname{sen}^{2} t \\
e_{2} \cos ^{2} t+2 f_{2} \cos t \operatorname{sen} t+g_{2} \operatorname{sen}^{2} t
\end{array}\right]
$$

$\mathrm{e}$

$$
H(p)=\left[\begin{array}{c}
\frac{e_{1}+g_{1}}{2} \\
\frac{e_{2}+g_{2}}{2}
\end{array}\right],
$$


onde, nas expressões acima, aparecem as componentes com relação ao referencial ortonormal $\left\{N_{1}, N_{2}\right\}$ de $N_{p} M$. Utilizando as identidades $\cos 2 t=\cos ^{2} t-\operatorname{sen}^{2} t$ e $\operatorname{sen} 2 t=2 \operatorname{sen} t \cos t$, podemos rescrever $\eta(p)(w)$ da seguinte forma

$$
\eta(p)(w)=\left[\begin{array}{ll}
\frac{1}{2}\left(e_{1}-g_{1}\right) & f_{1} \\
\frac{1}{2}\left(e_{2}-g_{2}\right) & f_{2}
\end{array}\right] \cdot\left[\begin{array}{l}
\cos 2 t \\
\operatorname{sen} 2 t
\end{array}\right]+\left[\begin{array}{c}
\frac{e_{1}+g_{1}}{2} \\
\frac{e_{2}+g_{2}}{2}
\end{array}\right],
$$

donde,

$$
\eta(p)(w)-H(p)=\left[\begin{array}{ll}
\frac{1}{2}\left(e_{1}-g_{1}\right) & f_{1} \\
\frac{1}{2}\left(e_{2}-g_{2}\right) & f_{2}
\end{array}\right] \cdot\left[\begin{array}{l}
\cos 2 t \\
\operatorname{sen} 2 t
\end{array}\right] .
$$

Podemos destacar os seguintes resultados (veja figura 2.1.1):

i) Como $\eta(p): S^{1} \subset T_{p} M \rightarrow N_{p} M$ é afim, segue que sua imagem descreve uma elipse em $N_{p} M$, chamada a elipse de curvatura de $\alpha$ no ponto $p$ e denotada por $\varepsilon_{\alpha}(p)$;

ii) Esta elipse pode se degenerar em um segmento de reta, uma circunferência ou um ponto;

iii) $\mathrm{O}$ centro da elipse de curvatura é o vetor curvatura média $H$. Portanto, a elipse de curvatura $\varepsilon_{\alpha}(p)$ pode ser escrita como $(\eta-H)+H$;

iv) A área da elipse de curvatura $\varepsilon_{\alpha}(p)$ é calculada como sendo a área de $S^{1}$ 
multiplicada pelo módulo do determinante da transformação afim, ou seja,

$$
\pi\left|\frac{1}{2}\left(e_{1}-g_{1}\right) f_{2}+\frac{1}{2}\left(g_{2}-e_{2}\right) f_{1}\right|=\frac{\pi}{2}\left|k_{N}(p)\right| ;
$$

v) A aplicação $\eta(p)$ restrita a $S^{1}$, sendo quadrática, é um duplo recobrimento da elipse de curvatura $\varepsilon_{\alpha}(p)$. Assim, todo ponto da elipse corresponde a dois pontos diametralmente opostos na circunferência tangente unitária, aos quais faremos corresponder uma direção em $T_{p} M$;

vi) A elipse de curvatura é invariante por rotações nos planos tangente e normal, logo podemos olhar a sua área, a curvatura normal, e o seu centro, o vetor curvatura média, como invariantes da imersão $\alpha$. Não é difícil observar que a resultante $\Delta$ também é um invariante da imersão.
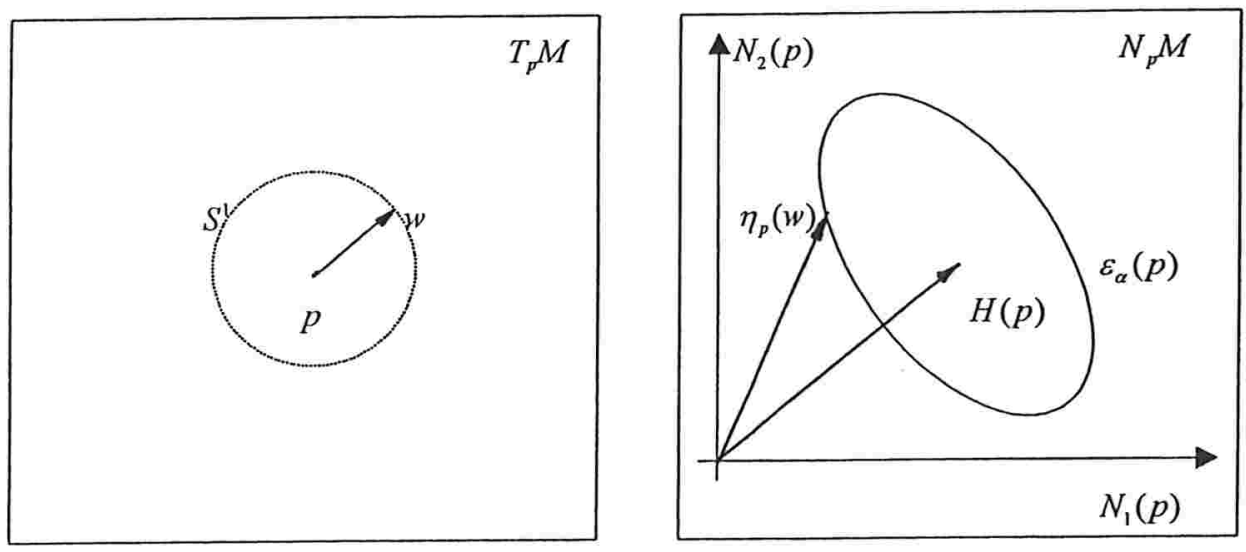

Figura 2.1.1. A elipse de curvatura.

Um ponto $p \in M$ é chamado de ponto mínimo de $\alpha$ se $H(p)=0$, e é 
chamado de ponto de inflexão de $\alpha$ se $\Delta(p)=k_{N}(p)=0$. Segue que $p$ é um ponto de inflexão se, e somente se,

$$
e_{1} f_{2}-e_{2} f_{1}=e_{1} g_{2}-e_{2} g_{1}=f_{1} g_{2}-f_{2} g_{1}=0
$$

ou por iv), se, e somente se, a elipse de curvatura $\varepsilon_{\alpha}(p)$ for um segmento de reta radial.

Segue de v) que a partir de qualquer bem definida escolha de pontos sobre a elipse, campos de direções tangentes podem ser construídos sobre $M$. Se a escolha não está bem definida para pontos especiais de $M$, dizemos que tais pontos são pontos singulares do campo de direções.

Consideremos alguns exemplos destas construções.

Campos de direções assintóticas. Suponhamos que a origem de $N_{p} M$ não pertença à região limitada pela elipse de curvatura $\varepsilon_{\alpha}(p), \forall p \in M$. Os dois pontos sobre $\varepsilon_{\alpha}(p)$ nos quais as retas geradas pelos vetores curvatura normal são tangentes a $\varepsilon_{\alpha}(p)$ induzem um par de direções no $T_{p} M$, às quais, em geral, não são ortogonais. Fazendo esta construção para todo $p \in M$, temos bem definidos dois campos de direções tangentes sobre $M$, chamados campos de direções assintóticas. As singularidades destes campos são os pontos onde a elipse de curvatura se torna um segmento de reta radial, ou seja, os pontos de inflexão, conforme observação acima. Garcia, Mochida, Fuster e Ruas estudaram estes campos e suas singularidades em [GMFR]. Veja figura 2.1.2.

Na figura 2.1.2 as retas geradas pelo vetor curvatura normal são tangentes à elipse de curvatura nos pontos 1 e 2 . As pré-imagens do ponto 1 são os pontos $1^{\prime}$ e $1^{\prime \prime}$ em $S^{1}(p) \subset T_{p} M$, os quais determinam a reta $1^{\prime}-1^{\prime \prime}$. De modo análogo o 
ponto 2 induz a reta $2^{\prime}-2^{\prime \prime}$. Como, de um modo geral, os pontos 1 e 2 não são simétricos com relação ao centro da elipse segue que as retas $1^{\prime}-1^{\prime \prime}$ e $2^{\prime}-2^{\prime \prime}$ não são ortogonais, de um modo geral. No capítulo 6 veremos um caso onde isto não acontece, ou seja, ocorre a ortogonalidade dos campos de direções assintóticas.
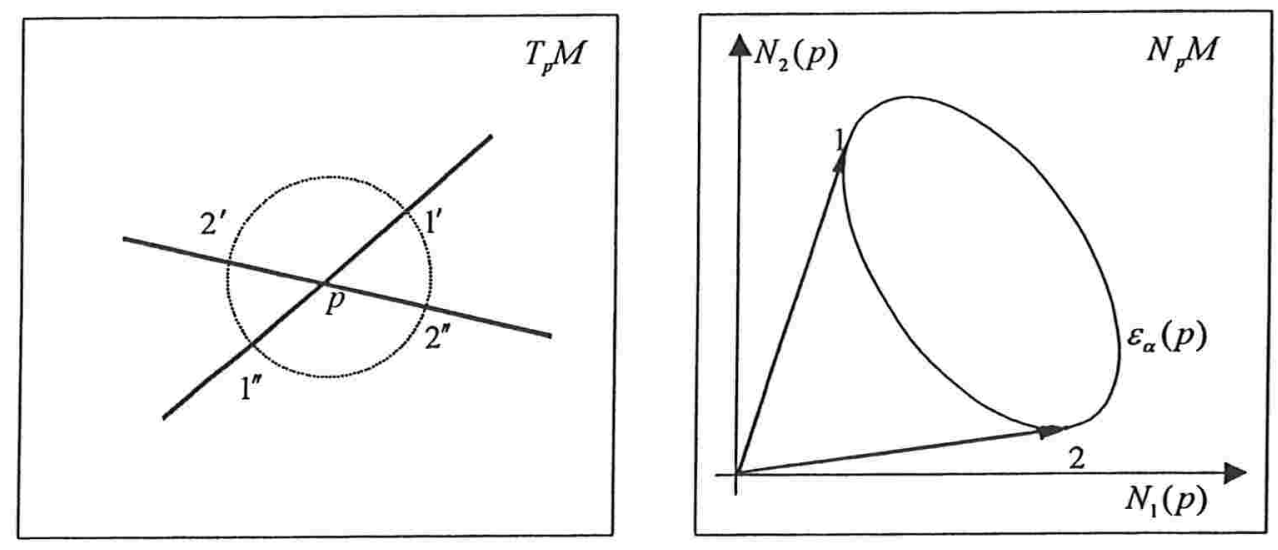

Figura 2.1.2. Campos de direções assintóticas.

Campos de direções de curvatura axial. Os dois máximos e os dois mínimos da norma de $(\eta-H)(p)$ determinam quatro pontos sobre a elipse de curvatura $\varepsilon_{\alpha}(p)$, os quais são seus vértices. Esta construção induz oito pontos sobre a circunferência $S^{1} \subset T_{p} M$, os quais definem quatro direções no plano tangente. Fazendo esta construção para todo $p \in M$ definimos um campo de 2-cruzes sobre $M$, chamados campos de direções de curvatura axial. Esta construção falha nos pontos axiumbílicos, onde a elipse de curvatura se torna uma circunferência ou um ponto. Gutierrez, Guadalupe, Tribuzy e Guíñez em [GGTG] e Garcia e Sotomayor em [GaS1] estudaram este caso. Veja figura 2.1.3.

Na figura 2.1.3 os vértices da elipse de curvatura estão denotados pelos 
números 1, 2, 3 e 4 . Os vértices 1 e 3 determinam o eixo menor da elipse, enquanto os vértices 2 e 4 determinam o eixo maior da elipse de curvatura. $\mathrm{O}$ ponto 1 da elipse de curvatura induz a direção $1^{\prime}-1^{\prime \prime}$, o ponto 2 a direção $2^{\prime}-2^{\prime \prime}$, o ponto 3 a direção $3^{\prime}-3^{\prime \prime}$ e o ponto 4 a direção $4^{\prime}-4^{\prime \prime}$. Como os pontos 1 e 3 são simétricos com relação ao centro da elipse, segue que as direções $1^{\prime}-1^{\prime \prime}$ e $3^{\prime}-3^{\prime \prime}$ são ortogonais. O mesmo sendo válido para os pontos 2 e 4 e para as direções $2^{\prime}-2^{\prime \prime}$ e $4^{\prime}-4^{\prime \prime}$. Agora, como o ponto 2 da elipse é "médio" dos pontos 1 e 3 , segue que a direção $2^{\prime}-2^{\prime \prime}$ bissecta as direções $1^{\prime}-1^{\prime \prime}$ e $3^{\prime}-3^{\prime \prime}$. O mesmo raciocínio é válido para o ponto 4 e para a direção $4^{\prime}-4^{\prime \prime}$.
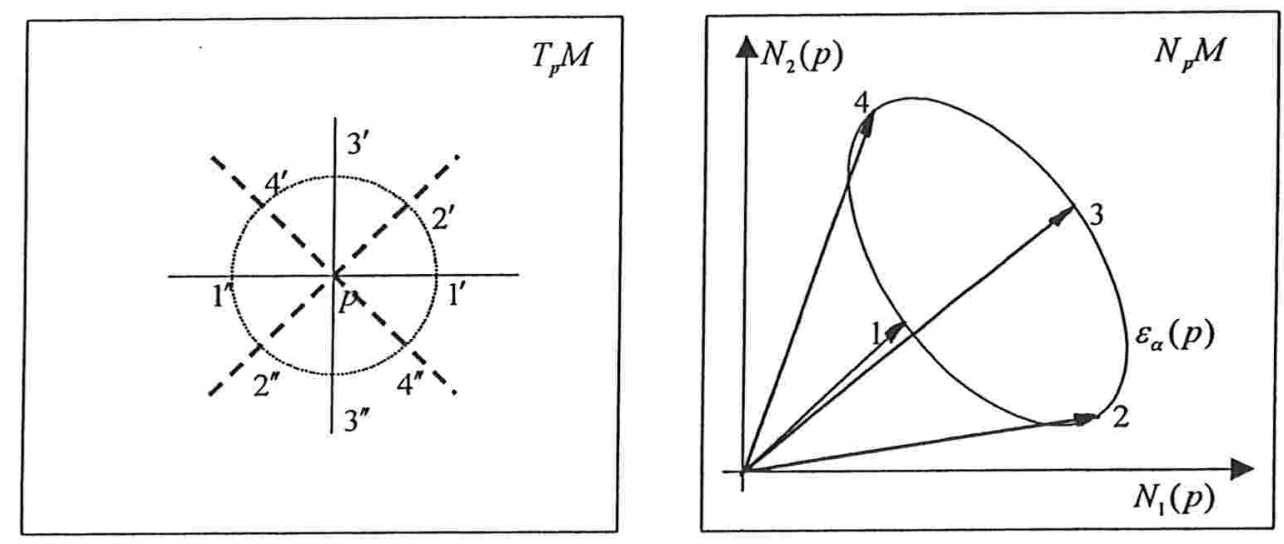

Figura 2.1.3. Campos de direções de curvatura axial.

Campos de direções de curvatura direcional média. A reta gerada pelo vetor curvatura média $H(p)$ intercepta a elipse de curvatura $\varepsilon_{\alpha}(p)$ em dois pontos. Esta construção induz duas direções ortogonais em $T_{p} M$. Fazendo esta construção para todo $p \in M$ definimos dois campos de direções sobre $M$, chamados campos de direções de curvatura direcional média. As singularidades destes campos, chamadas aqui $H$-singularidades, são os pontos onde a construção acima falha, ou 
seja, onde $H=0$ (pontos mínimos) ou onde a elipse de curvatura se torna um segmento de reta radial (pontos de inflexão). O conjunto das $H$-singularidades será denotado por $S(\alpha)$. Veja figura 2.1.4.

Na figura 2.1.4 os pontos 1 e 2 representam os pontos sobre a elipse de curvatura resultado da interseção desta com a reta gerada pelo vetor curvatura média $H$. O ponto 1 induz a direção $1^{\prime}-1^{\prime \prime}$, enquanto o ponto 2 induz a direção $2^{\prime}-2^{\prime \prime}$ sobre $T_{p} M$. Como os pontos 1 e 2 são simétricos com relação ao centro da elipse segue que as direções acima são ortogonais.
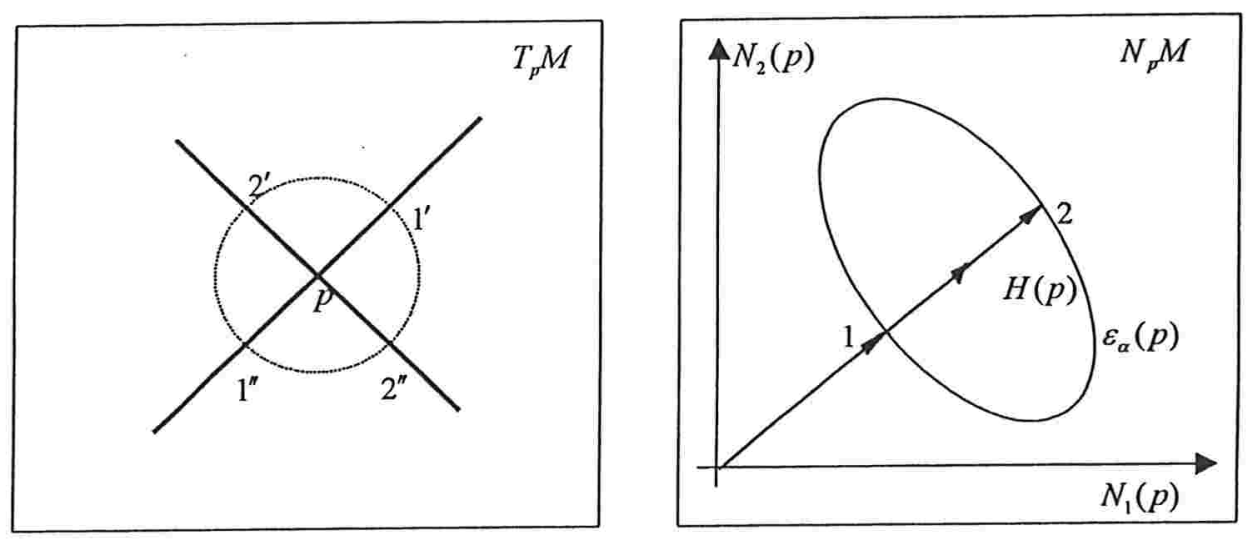

Figura 2.1.4. Campos de direções de curvatura direcional média.

Uma linha de curvatura direcional média é uma curva regular $\varphi:(a, b) \rightarrow M$ tal que em cada um de seus pontos a reta tangente a ela está numa direção de curvatura direcional média, e além disto ela contém qualquer outra curva regular com esta propriedade e que a intercepte. 


\subsection{A Equação Diferencial das Linhas de Curvatura Direcional Média}

Na definição das direções de curvatura direcional média exigimos que os vetores $H$ e $\eta$ sejam paralelos, assim a equação diferencial das linhas de curvatura direcional média é dada por

$$
\eta \wedge H=0,
$$

a qual é uma equação diferencial quadrática da forma

$$
A(u, v) d u^{2}+2 B(u, v) d u d v+C(u, v) d v^{2}=0,
$$

onde

$$
\begin{aligned}
& A=A(u, v)=\left(e_{1} g_{2}-e_{2} g_{1}\right) E+2\left(e_{2} f_{1}-e_{1} f_{2}\right) F, \\
& B=B(u, v)=\left(f_{1} g_{2}-f_{2} g_{1}\right) E+\left(e_{2} f_{1}-e_{1} f_{2}\right) G
\end{aligned}
$$

e

$$
C=C(u, v)=2\left(f_{1} g_{2}-f_{2} g_{1}\right) F+\left(e_{2} g_{1}-e_{1} g_{2}\right) G .
$$

As $H$-singularidades são determinadas através de (2.2.2), sendo caracterizadas como os pontos onde $A=B=C=0$. No entanto, é imediato a verificação de que

$$
E C=2 F B-G A .
$$

Temos estabelecido a seguinte proposição.

Proposição 2.2.1. Seja $\alpha: M \rightarrow R^{4}$ uma imersão de classe $C^{r}, r \geq 3$, de uma superfície regular e orientada $M \mathrm{em} R^{4}$. Com as notações acima temos:

(i) A equação diferencial das linhas de curvatura direcional média de $\alpha$ é dada por (2.2.2); 
(ii) As $H$-singularidades de $\alpha$ são dadas por $A=B=0$, onde $A$ e $B$ estão definidos em (2.2.3) e em (2.2.4), respectivamente.

Notemos que a equação diferencial das linhas de curvatura direcional média (2.2.2) pode ser escrita, equivalentemente, como

$$
\operatorname{Jac}\left\{\operatorname{Jac}\left(I_{1}, I_{2}\right), I\right\}=0 \text {. }
$$

Em coordenadas isotérmicas, onde $E=G=\lambda^{2}$ e $F=0$, a equação (2.2.2) tem a forma

$$
A_{1}(u, v) d u^{2}+2 B_{1}(u, v) d u d v-A_{1}(u, v) d v^{2}=0,
$$

onde

$$
A_{1}=e_{1} g_{2}-e_{2} g_{1}
$$

$\mathrm{e}$

$$
B_{1}=f_{1}\left(e_{2}+g_{2}\right)-f_{2}\left(e_{1}+g_{1}\right) \text {. }
$$

Lema 2.2.2. $A(p)=B(p)=0$ se, e somente se, $H(p)=0$ ou $p$ é um ponto de inflexão de $M$.

Prova. É suficiente provar o lema para o caso das coordenadas isotérmicas. Façamos

e

$$
0=A_{1} f_{1}+B_{1} g_{1}=\left(e_{1}+g_{1}\right)\left(f_{1} g_{2}-f_{2} g_{1}\right)
$$

$$
0=A_{1} f_{2}+B_{1} g_{2}=\left(e_{2}+g_{2}\right)\left(f_{1} g_{2}-f_{2} g_{1}\right) \text {. }
$$

$\mathrm{Se}$

então

$$
f_{1} g_{2}-f_{2} g_{1} \neq 0
$$

$$
e_{1}+g_{1}=e_{2}+g_{2}=0 \text {, }
$$

e isto implica que $H(p)=0$. Se 
então

$$
f_{1} g_{2}-f_{2} g_{1}=0
$$

$$
e_{1} g_{2}-e_{2} g_{1}=f_{1} g_{2}-f_{2} g_{1}=e_{1} f_{2}-e_{2} f_{1}=0
$$

e isto implica que $p$ é um ponto de inflexão. A recíproca é imediata.

Observação 2.2.3. A partir do lema 2.2.2 segue que $S(\alpha)=S_{1}(\alpha) \cup S_{2}(\alpha)$, onde

$$
S_{1}(\alpha)=\{p \in M / H(p)=0\}
$$

e

$$
S_{2}(\alpha)=\{p \in M / p \text { é um ponto de inflexão }\} \text {. }
$$

Aqui cabe um pequeno comentário a respeito da natureza dos conjuntos acima. Pode parecer a primeira vista que a separação de $S(\alpha)$ em dois conjuntos seja artificial, ou seja, os conjuntos $S_{1}(\alpha)$ e $S_{2}(\alpha)$ teriam apenas uma roupagem diferente, sendo no fundo os mesmos. Isto, de fato, não acontece. Conforme veremos no capítulo 8, existem imersões de superfícies em $R^{4}$ exibindo somente um tipo de $H$-singularidade. A posteriori, capítulo 8 , podemos perceber, por exemplo, a não possibilidade de construirmos um campo de direções sobre uma superficie imersa em $R^{4}$ que exiba apenas singularidades nos pontos mínimos.

Seja $p \notin S(\alpha)$. Segue que $\left(B^{2}-A C\right)(p)>0$, o que implica na existência de duas soluções ortogonais para a equação diferencial das linhas de curvatura direcional média (2.2.2). Assim, numa vizinhança deste ponto existem dois campos de direções, denotados por $L_{H}(\alpha)$ e $l_{H}(\alpha)$, o que, por sua vez, implica na existência de duas famílias de curvas ortogonais, denotadas também por $L_{H}(\alpha)$ e $l_{H}(\alpha)$. Sob as hipóteses de orientabilidade, é possível globalizar para toda a superfície $M$ a definição dos campos de direções acima, o que fazemos a seguir.

Observemos que em $M-S(\alpha)$ a equação (2.2.2) define um campo de 
cones ortogonais. Para o que segue consideraremos apenas os setores não negativos de cada cone, conforme figura 2.2.1.

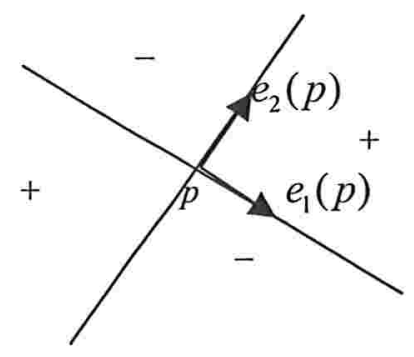

Figura 2.2.1. O cone em $p$.

Para cada $p \in M-S(\alpha)$ tomemos $\left\{e_{1}(p), e_{2}(p)\right\}$ consistindo de vetores unitários das duas direções de curvatura direcional média, de tal sorte que este conjunto seja uma base positiva de $T_{p} M$ e positiva também para o cone em $p$, isto é, o setor compreendido entre $e_{1}(p)$ e $e_{2}(p)$ é positivo. Podemos agora definir $L_{H}(\alpha)=$ R. $e_{1}$ e $l_{H}(\alpha)=R \cdot e_{2}$. Deste modo, estes dois campos de direções estão globalmente definidos, o que implica que as duas famílias de curvas ortogonais $L_{H}(\alpha)$ e $l_{H}(\alpha)$ também estão globalmente definidas, e recebem o nome de linhas de curvatura direcional média máxima e linhas de curvatura direcional média mínima, respectivamente. Cada família define uma folheação, a folheação direcional média máxima $F_{H}(\alpha)$ e a folheação direcional média mínima $f_{H}(\alpha)$, na superfície fora das $H$-singularidades. Cada $H$-singularidade isolada define uma singularidade isolada de ambas as folheações.

Podemos dar uma explicação a respeito dos nomes máximo e mínimo utilizados acima. Para qualquer ponto $p \notin S(\alpha)$, sejam $p_{1}(p)$ e $p_{2}(p)$ os dois pontos no plano normal nos quais a reta gerada por $H(p)$ intercepta $\varepsilon_{\alpha}(p)$. 
Genericamente temos $p_{1}(p) \neq p_{2}(p)$, pois, $p_{1}(p)=p_{2}(p)$ ocorre apenas quando a elipse de curvatura é um segmento de reta, ou seja, quando $k_{N}(p)=0$. Suponhamos que $k_{N}(p)>0$. Neste caso, apenas um ponto, digamos $p_{2}(p)$, está mais afastado da origem. A direção de curvatura direcional média máxima (respectivamente mínima) é a direção de curvatura direcional média induzida por $p_{2}(p)$ (respectivamente $\left.p_{1}(p)\right)$. No caso em que $k_{N}(p)<0$, devemos trocar $p_{2}$ e $p_{1}$. 


\section{Capítulo 3. Estudo das H-Singularidades}

Seja $p$ uma $H$-singularidade de uma imersão $\alpha: M^{2} \rightarrow R^{4}$. Dizemos que $p$ é uma $H$-singularidade transversal se

$$
\left.J(A, B)\right|_{p}=\left.\frac{\partial(A, B)}{\partial(u, v)}\right|_{p} \neq 0,
$$

onde as funções $A$ e $B$ estão definidas em (2.2.3) e (2.2.4), respectivamente. O significado geométrico desta condição é que as curvas $A^{-1}(0)$ e $B^{-1}(0)$, cujas interseções definem as $H$-singularidades, são regulares e se interceptam transversalmente no ponto $p$. Segue que as $H$-singularidades transversais são isoladas.

Recordemos que o índice $i(p)$ de um campo de direções em uma singularidade isolada $p$ é o número total de voltas que o campo executa depois de percorrer uma vez o bordo de um disco, positivamente orientado, contendo a singularidade em seu interior (veja [Hop]). Seja $p$ uma $H$-singularidade isolada. Então $p$ é uma singularidade isolada de cada uma das duas famílias de linhas de curvatura direcional média. Deste modo $p$ tem um índice com respeito a cada uma destas famílias; mas, uma vez que as linhas de uma família são ortogonais às linhas da outra família, segue que estes índices devem ser iguais. 
Seja $X=\left(X_{1}, X_{2}\right)$ um campo de vetores planar e seja $p$ uma singularidade isolada de $X$. Dizemos que $p$ é uma singularidade transversal de $X$ se $J(X(p)) \neq 0$. Novamente, o significado geométrico desta condição é que as curvas $X_{1}^{-1}(0)$ e $X_{2}^{-1}(0)$, cujas interseções definem as singularidades de $X$, são regulares e se interceptam transversalmente no ponto $p$.

Neste caso, o índice (do campo $X$ ) na singularidade $p$ é dado por

$$
I(X, p)=\text { sinal de }\{J(X(p))\} .
$$

Usando as funções $A_{1}$ e $B_{1}$ definidas em (2.2.8) e (2.2.9), respectivamente, definimos o campo de vetores

$$
X(u, v)=\left(-B_{1}(u, v), A_{1}(u, v)\right)
$$

Assim, $p$ é uma $H$-singularidade isolada de $\alpha$ se, e somente se, $p$ for uma singularidade isolada do campo de vetores $X$.

Lema 3.1. Seja $i(p)$ o índice de uma $H$-singularidade isolada $p \in M$ e seja $I(X, p)$ o índice da singularidade isolada do campo de vetores $X$, como em (3.2). Então temos

$$
i(p)=\frac{1}{2} I(X, p) .
$$

Prova. Seja $p=(u, v)=u+i v$ uma $H$-singularidade isolada. Deste modo o elemento $(d u, d v)=d u+i d v$ resolve a equação (2.2.7) se, e somente se, o vetor

$$
\left(A_{1}(u, v), B_{1}(u, v)\right)
$$

for ortogonal a 


$$
\left(d u^{2}-d v^{2}, 2 d u d v\right)
$$

Equivalentemente, o vetor ortogonal a (3.4), o qual tem a forma

$$
\left(-B_{1}(u, v), A_{1}(u, v)\right)=X(u, v)
$$

for colinear a (3.5). Mas, (3.5) pode ser escrito como

$$
\left(d u^{2}-d v^{2}, 2 d u d v\right)=d u^{2}-d v^{2}+i 2 d u d v=(d u+i d v)^{2},
$$

em notação complexa. Como o argumento de $(d u+i d v)^{2}$ é o dobro do argumento de $d u+i d v$, o lema está provado.

Passaremos agora ao estudo dos dois tipos de $H$-singularidades transversais. Achamos mais conveniente fazê-lo separadamente. Deste modo podemos destacar as peculiaridades de cada caso, dando destaque ao caso das $H$ singularidades transversais que são pontos mínimos, ou seja, a $H$-singularidade transversal pertence ao conjunto $S_{1}(\alpha)$. Neste caso a $H$-singularidade transversal é também singularidade transversal do campo de vetores curvatura média $H$. Embora este campo não apresente dinâmica do ponto de vista da superfície, uma interessante relação entre o índice do campo $H$ e o índice do campo de direções de curvatura direcional média será estabelecido na seção 3.1. Na seção 3.2 estudaremos as $H$-singularidades transversais determinadas pelos pontos de inflexão, isto é, pertencentes a $S_{2}(\alpha)$.

\subsection{O Caso dos Pontos Mínimos}

Proposição 3.1.1. Seja $p \in M$ um ponto onde $H(p)=0$ e $k_{N}(p) \neq 0$. Então $p$ é uma singularidade transversal do campo de vetores $X$, como em (3.2), se, e 
somente se, $p$ é uma singularidade transversal do campo de vetores (normais) $H$.

Prova. Temos a seguinte equação

$$
\left[\begin{array}{cc}
\left(-B_{1}\right)_{u} & \left(-B_{1}\right)_{v} \\
\left(A_{1}\right)_{u} & \left(A_{1}\right)_{v}
\end{array}\right]_{p}=\left[\begin{array}{cc}
f_{2} & -f_{1} \\
g_{2} & e_{1}
\end{array}\right]_{p} \cdot\left[\begin{array}{cc}
\left(e_{1}+g_{1}\right)_{u} & \left(e_{1}+g_{1}\right)_{v} \\
\left(e_{2}+g_{2}\right)_{u} & \left(e_{2}+g_{2}\right)_{v}
\end{array}\right]_{p}
$$

Assim,

$$
\begin{aligned}
J(X(p)) & =\left(f_{2} e_{1}+f_{1} g_{2}\right)_{p} 4(E(p))^{2} J(H(p))= \\
& =\frac{1}{2}\left[f_{1}\left(g_{2}+g_{2}\right)+f_{2}\left(e_{1}+e_{1}\right)\right]_{p} 4(E(p))^{2} J(H(p)) .
\end{aligned}
$$

Como $H(p)=0$, segue que $e_{1}=-g_{1}$ e $e_{2}=-g_{2}$. Deste modo, temos

$$
\begin{aligned}
J(X(p)) & =2\left[f_{1}\left(g_{2}-e_{2}\right)+f_{2}\left(e_{1}-g_{1}\right)\right]_{p}(E(p))^{2} J(H(p))= \\
& =2 k_{N}(p)(E(p))^{4} J(H(p)) .
\end{aligned}
$$

Uma vez que $k_{N}(p) \neq 0$ e $E(p)>0$, a proposição está provada.

Teorema 3.1.2. Seja $p \in M$ uma $H$-singularidade, onde $H(p)=0$ e $k_{N}(p) \neq 0$. Então $p$ é uma $H$-singularidade transversal se, e somente se, $p$ é uma singularidade transversal do campo de vetores (normais) $H$.

Prova. É imediata a partir da proposição 3.1 .1 e de

$$
\left.\frac{\partial\left(A_{1}, B_{1}\right)}{\partial(u, v)}\right|_{p}=\operatorname{det}\left[\begin{array}{ll}
\left(A_{1}\right)_{u} & \left(A_{1}\right)_{v} \\
\left(B_{1}\right)_{u} & \left(B_{1}\right)_{v}
\end{array}\right]_{p}=\operatorname{det}\left[\begin{array}{cc}
\left(-B_{1}\right)_{u} & \left(-B_{1}\right)_{v} \\
\left(A_{1}\right)_{u} & \left(A_{1}\right)_{v}
\end{array}\right]_{p}=J(X(p))
$$


Teorema 3.1.3. Seja $p \in M$ um ponto onde $H(p)=0$ e $k_{N}(p) \neq 0$. Suponhamos que $p$ seja uma $H$-singularidade transversal. Então, o índice do campo de vetores curvatura média $H$ em $p, I(H, p)$, é \pm 1 . E mais, o índice da $H$-singularidade $p$, $i(p)$, é $\pm 1 / 2$. Os sinais concordam se $k_{N}(p)>0$, e discordam se $k_{N}(p)<0$.

Prova. Como $p$ é uma $H$-singularidade transversal, segue do teorema 3.1 .2 e de (3.1) que

$$
I(H, p)=\text { sinal de }\{J(H(p))\}= \pm 1 .
$$

De (3.1.2), temos

$$
J(X(p))=2 k_{N}(p)(E(p))^{4} J(H(p)) .
$$

Assim,

$$
\text { sinal de }\{J(X(p))\}=\left(\operatorname{sinal} \operatorname{de}\left(k_{N}(p)\right)\right) .(\text { sinal de }\{J(H(p))\}) .
$$

Isto implica que

$$
I(X, p)=\left(\operatorname{sinal} \operatorname{de}\left(k_{N}(p)\right)\right) \cdot I(H, p) .
$$

Mas de (3.3) e (3.1), temos

$$
\begin{aligned}
i(p) & =\frac{1}{2}\left(\operatorname{sinal} \operatorname{de}\left(k_{N}(p)\right)\right) \cdot I(H, p)=\frac{1}{2}\left(\operatorname{sinal} \operatorname{de}\left(k_{N}(p)\right)\right) \cdot( \pm 1)= \\
& = \pm \frac{1}{2} \operatorname{sinal~de}\left(k_{N}(p)\right) .
\end{aligned}
$$

Este teorema aparece, com uma outra demonstração, em [Lit]. No entanto, há lá um engano com relação à interpretação dos sinais dos dois índices e o sinal da curvatura normal. Asperti, em [Asp] fez a correção deste engano, utilizando as mesmas técnicas de Little, diferentes das aqui apresentadas. 


\subsection{O Caso dos Pontos de Inflexão}

Definimos as seguintes funções

$$
T_{1}=e_{1} g_{2}-e_{2} g_{1}, T_{2}=f_{1} g_{2}-f_{2} g_{1} \text { e } T_{3}=e_{1} f_{2}-e_{2} f_{1}
$$

e os seguintes campos de vetores

$$
Y_{1}(u, v)=\left(T_{1}(u, v), T_{2}(u, v)\right)
$$

$\mathrm{e}$

$$
Y_{2}(u, v)=\left(T_{1}(u, v), T_{3}(u, v)\right)
$$

Notemos que $T_{1}(p)=T_{2}(p)=T_{3}(p)=0$ se, e somente se, $p$ é um ponto de inflexão, donde $Y_{1}(p)=Y_{2}(p)=0$ se, e somente se, $p$ é um ponto de inflexão.

Proposição 3.2.1. Seja $p \in M$ um ponto de inflexão onde $H(p) \neq 0$. Então, $p$ é uma singularidade transversal do campo de vetores $X$ em (3.2) se, somente se, $p$ é uma singularidade transversal do campo de vetores $Y_{1}$.

Prova. Temos a seguinte equação

$$
J(X(p))=J\left(Y_{1}(p)\right)-J\left(Y_{2}(p)\right) .
$$

Mas,

$$
J\left(Y_{1}(p)\right)=-J\left(Y_{2}(p)\right) .
$$

De (3.2.4) e de (3.2.5) segue que

$$
J(X(p))=2 J\left(Y_{1}(p)\right) .
$$

Teorema 3.2.2. Seja $p \in M$ um ponto de inflexão onde $H(p) \neq 0$. Então, $p$ é uma $H$-singularidade transversal se, e somente se, $p$ é uma singularidade 
transversal do campo de vetores $Y_{1}$.

Prova. Imediata a partir da proposição 3.2 .1 e de

$$
\left.\frac{\partial\left(A_{1}, B_{1}\right)}{\partial(u, v)}\right|_{p}=J(X(p)) .
$$

Teorema 3.2.3. Seja $p \in M$ um ponto de inflexão onde $H(p) \neq 0$. Suponhamos que $p$ seja uma $H$-singularidade transversal. Então, o índice da $H$-singularidade $p$, $i(p)$, é $\pm 1 / 2$.

Prova. De (3.2.6) e de (3.1), temos

$$
I(X, p)=\text { sinal de }\{J(X(p))\}=\text { sinal de }\left\{J\left(Y_{1}(p)\right)\right\}= \pm 1 .
$$

Utilizando (3.3), segue que

$$
i(p)=\frac{1}{2} I(X, p)= \pm \frac{1}{2} .
$$

Observação 3.2.4. Em outras palavras, o que os resultados acima estão dizendo é que, embora na caracterização dos pontos de inflexão façamos uso das três funções $T_{1}, T_{2}$ e $T_{3}$, apenas duas delas, por exemplo, $T_{1}$ e $T_{2}$ são, de fato, essenciais. A interpretação geométrica da transversalidade de um ponto de inflexão é a mesma das anteriores, ou seja, $p$ é um ponto de inflexão transversal se, e somente se, as curvas $T_{1}^{-1}(0)$ e $T_{2}^{-1}(0)$ são regulares e se interceptam transversalmente no ponto $p$.

Os resultados desta seção serão utilizados também no capítulo 6 , onde apresentamos uma outra expressão para a equação diferencial das linhas 
assintóticas, diferente daquela que aparece em [GMFR]. 


\section{Capítulo 4. H-Singularidades do Tipo $S$}

No capítulo anterior estudamos as $H$-singularidades transversais e vimos que elas são caracterizadas pela transversalidade das curvas que as definem. Neste capítulo estudaremos um subconjunto das $H$-singularidades transversais, o qual, como veremos mais adiante (capítulo 7), tem a propriedade de ser denso no conjunto das $H$-singularidades. Podemos ainda adiantar uma outra propriedade das $H$-singularidades deste conjunto, elas são, num certo sentido a ser precisado (capítulo 7), localmente estáveis. Por ora basta a idéia intuitiva de estabilidade que todos nós temos. Voltaremos a falar nisso mais adiante.

Vamos tomar a superfície $M$ na carta de Monge, isto é, a superfície $M$ é o gráfico da aplicação

$$
\alpha(u, v)=(u, v, S(u, v), R(u, v))
$$

onde $S$ e $R$ são funções de classe $C^{r}, r \geq 4$, definidas em uma vizinhança $U \subset R^{2}$ de $(0,0)$, satisfazendo

$$
S(0,0)=R(0,0)=S_{u}(0,0)=R_{u}(0,0)=S_{v}(0,0)=R_{v}(0,0)=0 .
$$

Para cada ponto $\alpha(u, v) \in M$ o plano tangente $T_{\alpha(u, v)} M$ é gerado por 


$$
\left\{\alpha_{u}(u, v)=\left(1,0, S_{u}(u, v), R_{u}(u, v)\right), \alpha_{v}(u, v)=\left(0,1, S_{v}(u, v), R_{v}(u, v)\right)\right\},
$$

bem como o plano normal $N_{\alpha(u, v)} M$ é gerado por

$$
\left\{\begin{array}{c}
\bar{N}_{1}=\left(-S_{u},-S_{v}, 1,0\right), \\
\bar{N}_{2}=\left(-R_{u}\left(1+S_{v}{ }^{2}\right)+S_{u} S_{v} R_{v},-R_{v}\left(1+S_{u}{ }^{2}\right)+S_{u} S_{v} R_{u},-S_{u} R_{u}-S_{v} R_{v}, 1+S_{u}{ }^{2}+S_{v}{ }^{2}\right)
\end{array}\right\} .
$$

Deste modo,

$$
\begin{gathered}
E=\left\langle\alpha_{u}, \alpha_{u}\right\rangle, F=\left\langle\alpha_{u}, \alpha_{v}\right\rangle, G=\left\langle\alpha_{v}, \alpha_{v}\right\rangle, \\
e_{i}=\left\langle\alpha_{u u}, N_{i}\right\rangle, f_{i}=\left\langle\alpha_{u v}, N_{i}\right\rangle \text { e } g_{i}=\left\langle\alpha_{v v}, N_{i}\right\rangle,
\end{gathered}
$$

onde

$$
N_{i}=\bar{N}_{i} /\left|\bar{N}_{i}\right| \text {, para } i=1,2 \text {. }
$$

Escrevendo a expansão de Taylor das funções $S$ e $R$ em torno de $(0,0)$, temos

$$
\begin{aligned}
& S(u, v)=\frac{s_{20}}{2} u^{2}+s_{11} u v+\frac{s_{02}}{2} v^{2}+\frac{a}{6} u^{3}+\frac{d}{2} u^{2} v+\frac{b}{2} u v^{2}+\frac{c}{6} v^{3}+O(4) \\
& R(u, v)=\frac{r_{20}}{2} u^{2}+r_{11} u v+\frac{r_{02}}{2} v^{2}+\frac{\bar{a}}{6} u^{3}+\frac{\bar{d}}{2} u^{2} v+\frac{\bar{b}}{2} u v^{2}+\frac{\bar{c}}{6} v^{3}+O(4) .
\end{aligned}
$$

Assim os coeficientes da primeira e da segunda formas fundamentais, na carta de Monge, são dados por

$$
\begin{gathered}
E=1+O(2), F=O(2), G=1+O(2), \\
e_{1}=s_{20}+a u+d v+O(2), f_{1}=s_{11}+d u+b v+O(2), \\
g_{1}=s_{02}+b u+c v+O(2), e_{2}=r_{20}+\bar{a} u+\bar{d} v+O(2), \\
f_{2}=r_{11}+\bar{d} u+\bar{b} v+O(2) \text { e } g_{2}=r_{02}+\bar{b} u+\bar{c} v+O(2) .
\end{gathered}
$$


Definimos as seguintes funções

$$
\begin{gathered}
J=s_{02} r_{20}-s_{20} r_{02}, \\
K=s_{02} \bar{a}+r_{20} b-s_{20} \bar{b}-r_{02} a, \\
L=s_{02} \bar{d}+r_{20} c-s_{20} \bar{c}-r_{02} d, \\
M=s_{11}\left(r_{20}+r_{02}\right)-r_{11}\left(s_{20}+s_{02}\right), \\
N=s_{11}(\bar{a}+\bar{b})-r_{11}(a+b)+\left(r_{20}+r_{02}\right) d-\left(s_{20}+s_{02}\right) \bar{d}
\end{gathered}
$$

e

$$
P=s_{11}(\bar{c}+\bar{d})-r_{11}(c+d)+\left(r_{20}+r_{02}\right) b-\left(s_{20}+s_{02}\right) \bar{b} .
$$

A equação diferencial das linhas de curvatura direcional média (2.2.2), escrita na carta de Monge, tem a forma

$$
C(u, v) d v^{2}+2 B(u, v) d u d v+A(u, v) d u^{2}=0,
$$

onde

$$
\begin{aligned}
& C(u, v)=J+K u+L v+Q_{1}(u, v), \\
& B(u, v)=M+N u+P v+Q_{2}(u, v), \\
& A(u, v)=-J-K u-L v+Q_{3}(u, v), \\
& Q_{1}, Q_{2} \text { e } Q_{3} \text { são da ordem } O(2) .
\end{aligned}
$$

No ponto $(0,0)$, temos

$$
\begin{gathered}
\alpha_{u}(0,0)=(1,0,0,0), \alpha_{v}(0,0)=(0,1,0,0), \\
N_{1}(0,0)=(0,0,1,0), N_{2}(0,0)=(0,0,0,1), \\
E(0,0)=G(0,0)=1, F(0,0)=0,
\end{gathered}
$$




$$
\begin{aligned}
& e_{1}(0,0)=s_{20}, f_{1}(0,0)=s_{11}, g_{1}(0,0)=s_{02}, \\
& e_{2}(0,0)=r_{20}, f_{2}(0,0)=r_{11} \text { e } g_{2}(0,0)=r_{02} .
\end{aligned}
$$

De (4.4), as condições para $(0,0)$ ser uma $H$-singularidade são

$$
J=s_{20} r_{02}-s_{02} r_{20}=0
$$

e

$$
M=s_{11}\left(r_{20}+r_{02}\right)-r_{11}\left(s_{20}+s_{02}\right)=0 .
$$

Seja $T M-\{0\}$ o fibrado tangente, onde removemos a seção nula, munido da relação $\sim$, definida da seguinte maneira: $(p, v) \sim(q, w) \Leftrightarrow p=q$ e $v=\lambda w, \lambda \neq 0$. É imediato que a relação $\sim$ é uma relação de equivalência em $T M-\{0\}$. Chamamos de fibrado projetivo de $M, P M$, o espaço quociente $T M-\{0\} / \sim$. A projeção natural será denotada por $\pi: P M \rightarrow M$.

Seja $p \in M$ uma $H$-singularidade. Então, existe uma vizinhança $V_{p} \subset M$ a qual pode ser descrita na carta de Monge. Nestas condições, a equação diferencial das linhas de curvatura direcional média é da forma dada em (4.4).

O espaço $P M$ pode ser parametrizado por duas cartas coordenadas $\left(u, v ; q=\frac{d u}{d v}\right)$ e $\left(u, v ; p=\frac{d v}{d u}\right)$. A equação diferencial das linhas de curvatura direcional média (4.4) define uma superfície $W$ no espaço $P M$. Na carta $(u, v ; p)$, a superfície $W$ é definida por $W=T^{-1}(0)$, onde

$$
T=\left(J+K u+L v+Q_{1}\right) p^{2}+2\left(M+N u+P v+Q_{2}\right) p-\left(J+K u+L v+Q_{3}\right) \text {. }
$$


Fora de $\pi^{-1}(0,0)$ a superfície $W$ é regular, sendo, de fato, um duplo recobrimento de $\pi^{-1}(M-\{(0,0)\})$. Além disto, a reta projetiva $p=\pi^{-1}(0,0)$ está contida em $W$. De fato,

$$
T(0,0, p)=J(0,0) p^{2}+2 M(0,0) p-J(0,0)=0
$$

se

$$
J(0,0)=M(0,0)=0,
$$

isto é, se $(0,0)$ for uma $H$-singularidade de $M$. Neste caso,

$$
\begin{gathered}
T_{u}(0,0, p)=K(0,0) p^{2}+2 N(0,0) p-K(0,0), \\
T_{v}(0,0, p)=L(0,0) p^{2}+2 P(0,0) p-L(0,0)
\end{gathered}
$$

e

$$
T_{p}(0,0, p)=2 J(0,0) p+2 M(0,0)=0 .
$$

Os polinômios $T_{u}(0,0, p) \mathrm{e} T_{v}(0,0, p)$ têm raiz comum se, e somente se,

$$
N(0,0) L(0,0)-K(0,0) P(0,0)=0,
$$

ou seja, os polinômios acima não têm raiz comum se, e somente se,

$$
N(0,0) L(0,0)-K(0,0) P(0,0) \neq 0,
$$

sendo esta última desigualdade uma condição necessária e suficiente para $(0,0)$ ser uma $H$-singularidade transversal de $M$. Pelo Teorema da Função Implícita, a superfície $W$ é regular numa vizinhança do eixo $p$ (reta projetiva) se, e somente se, $(0,0)$ for uma $H$-singularidade transversal.

Suponhamos que $(0,0)$ seja uma $H$-singularidade transversal. Neste caso podemos considerar o seguinte campo de vetores sobre $W$

$$
X(u, v, p)=\left(T_{p}(u, v, p), p T_{p}(u, v, p),-\left(T_{u}(u, v, p)+p T_{v}(u, v, p)\right)\right) .
$$


As seguintes afirmações são verificadas. Primeiramente, o campo $X$ é tangente a $W$. As singularidades de $\pi_{*}(X)$ ocorrem somente na origem, e mais, se $\left(u, v ; p_{j}\right) \in W$, então, $\pi_{*}\left(T\left(u, v ; p_{j}\right)\right)$ define uma linha de curvatura direcional média com direção $p_{j}$. As singularidades deste campo $X$, localizadas sobre a reta projetiva, são dadas pelas raízes do seguinte polinômio cúbico

$$
\varphi(p)=T_{u}(0,0, p)+p T_{v}(0,0, p)=0 .
$$

Uma direção $s \in T_{(0,0)} M$ é uma direção de aproximação da $H$ singularidade $(0,0)$ se existir uma linha de curvatura direcional média satisfazendo as seguintes duas condições:

i) contém a origem em seu fecho;

ii) o seu campo de direções tangente tem a direção $s$ como limite na $H$ singularidade.

Lema 4.1. Se $(0,0)$ é uma $H$-singularidade transversal, então, existe uma correspondência bijetiva entre as singularidades de $X$ ao longo da reta projetiva e as direções de aproximação de $(0,0)$.

Prova. Seja $v=p u+O(2)$ uma linha de curvatura direcional média. Então, $p$ é raiz de (4.7), donde $p$ é uma singularidade de $X$. Agora, se $(0,0, p)$ é uma singularidade de $X$, o vetor $(1, p, 0) \in T_{(0,0, p)} W$, uma vez que ele é normal ao gradiente de $W$ neste ponto. Deste modo, existe uma curva $\gamma$ em $W$ tal que seu vetor tangente em $(0,0, p)$ é $(1, p, 0)$ e $\pi(\gamma)$ é uma linha de curvatura direcional média. 
Uma separatriz da $H$-singularidade é a imagem não degenerada, sob $\pi_{*}$, de uma separatriz de sela do campo $X$. Elas são linhas de curvatura direcional média que tendem à $H$-singularidade e que separam regiões de diferentes modos de se aproximar da $H$-singularidade.

Como a cúbica (4.7) tem genericamente uma ou três raízes reais, o campo de vetores $X$ tem, genericamente, uma ou três singularidades sobre a reta projetiva, às quais são do tipo sela ou nó. Decorre ainda que temos apenas uma das seguintes três possibilidades:

i) O campo de vetores $X$ tem uma única singularidade do tipo sela. Neste caso $(0,0)$ é uma $H$-singularidade transversal do tipo $S_{1}$ (veja figura 4.1);

ii) $\mathrm{O}$ campo de vetores $X$ tem trềs singularidades, sendo um nó e duas selas. Neste caso $(0,0)$ é uma $H$-singularidade transversal do tipo $S_{2}$ (veja figura 4.1);

iii) $\quad \mathrm{O}$ campo de vetores $X$ tem três singularidades, todas do tipo sela. Neste caso $(0,0)$ é uma $H$-singularidade transversal do tipo $S_{3}$ (veja figura 4.1).

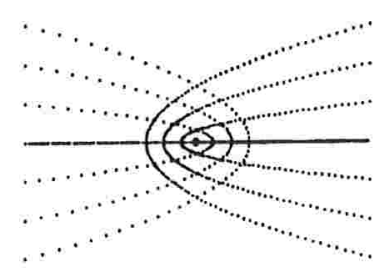

Tipo $S_{1}$

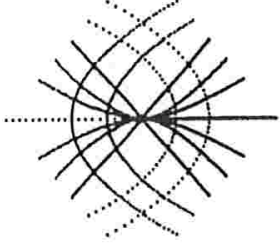

Tipo $S_{2}$

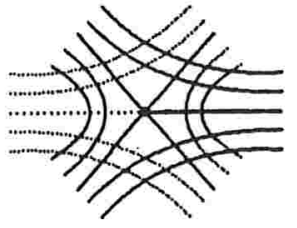

Tipo $S_{3}$

Figura 4.1. $H$-singularidades do tipo $S$. 
Observação 4.1. O índice $i=1,2,3$ de $S_{i}$ denota o número de separatrizes da $H$ singularidade. A figura 4.1 representa a configuração local dos três diferentes tipos de $H$-singularidades denotadas por $S_{1}, S_{2}$ e $S_{3}$ e chamadas de $H$-singularidades de tipo $S$. O índice é $1 / 2$ no caso $S_{1}$ e $S_{2}$, e $-1 / 2$ no caso $S_{3}$.

A seguir vamos analisar separadamente cada um dos dois casos de $H$ singularidades. Para cada um deles vamos dar uma descrição da superfície na carta de Monge, caracterizando os três tipos de $H$-singularidades em termos dos coeficientes dos 2 e 3 jatos da imersão.

\subsection{O Caso dos Pontos Mínimos}

Suponhamos que $p \in M$ seja uma $H$-singularidade satisfazendo as condições $H(p)=0$ e $k_{N}(p) \neq 0$. Escolhendo apropriadamente uma rotação no plano normal de modo que $N_{1}$ e $N_{2}$ estejam nas direções dos eixos principais da elipse de curvatura, é possível escrever $r_{20}=r_{02}=0$. Neste caso, decorre ainda que $s_{11}=0$. Agora, escolhendo apropriadamente uma rotação no plano $u v$ é possível escrever $\bar{b}=-\bar{a}$. Assim, (4.1) pode ser escrito como

$$
\begin{gathered}
S(u, v)=\frac{A}{2}\left(u^{2}-v^{2}\right)+\frac{a}{6} u^{3}+\frac{d}{2} u^{2} v+\frac{b}{2} u v^{2}+\frac{c}{6} v^{3}+O(4) \\
R(u, v)=C u v+\frac{\bar{a}}{6} u^{3}+\frac{\bar{d}}{2} u^{2} v-\frac{\bar{a}}{2} u v^{2}+\frac{\bar{c}}{6} v^{3}+O(4) .
\end{gathered}
$$

Isto implica que (4.3) tem a forma

$$
\begin{gathered}
J=0, K=0, L=-A(\bar{c}+\bar{d}), \\
M=0, N=-C(a+b) \text { e } P=-C(c+d) .
\end{gathered}
$$


Neste caso, o polinômio cúbico (4.7) tem a forma

$$
\varphi(p)=-p\left(L p^{2}+2 P p+2 N-L\right)
$$

Assim, $p=0$ é uma raiz e as outras irão depender do discriminante do polinômio quadrático entre parêntese, dado por $\Delta=\left(\frac{P}{L}\right)^{2}+1-\frac{2 N}{L}$. Se $\frac{2 N}{L}>\left(\frac{P}{L}\right)^{2}+1$, temos uma única raiz real em $p=0$. Se $\frac{2 N}{L}<\left(\frac{P}{L}\right)^{2}+1$, temos 3 raízes reais dadas por

$$
p_{0}=0, p_{1}=-\frac{P}{L}+\sqrt{\left(\frac{P}{L}\right)^{2}+1-\frac{2 N}{L}} \text { e } p_{2}=-\frac{P}{L}-\sqrt{\left(\frac{P}{L}\right)^{2}+1-\frac{2 N}{L}} .
$$

Notemos que, na hipótese de termos 3 raízes, $p_{1} \cdot p_{2}+1=\frac{2 N}{L}$. Assim, se $\frac{2 N}{L}<0$, então as direções $p_{1}$ e $p_{2}$ não estão em um setor de medida $\pi / 2 \cdot$ Se $\frac{2 N}{L}>0$, então as direções $p_{1}$ e $p_{2}$ estão em um setor de medida $\pi / 2$.

A matriz Jacobiana de $X$ em uma singularidade da reta projetiva é dado por

$$
\left[\begin{array}{ccc}
2 N & 2 L p+2 P & 0 \\
2 N p & p(2 L p+2 P) & 0 \\
* & * & -\left(3 L p^{2}+4 P p+2 N-L\right)
\end{array}\right]_{(0,0, p)}
$$

Podemos observar que o jacobiano de $X$ em $p=0$ é dado por 


$$
J X(0)=-2 N(2 N-L)
$$

Logo, $\frac{J X(0)}{L^{2}}=\left(\frac{2 N}{L}\right) \cdot\left(1-\frac{2 N}{L}\right)$. Podemos então tirar as seguintes conclusões:

i) Se $\Delta<0$, então $\frac{2 N}{L}>1$, o que implica que $J X(0)<0$, o que, por sua vez, implica que a origem é um ponto de sela.

ii) Se $1>\frac{2 N}{L}>0$, o que implica que $\Delta>0$, então $J X(0)>0$, o que implica que a origem é um nó. Analisando as outras duas raízes vemos que elas são pontos de sela.

iii) Se $\frac{2 N}{L}<0$, o que implica que $\Delta>0$, então $J X(0)<0$, o que implica que a origem é um ponto de sela. Analisando as outras duas raízes vemos que elas também são pontos de sela.

Definimos

$$
\begin{aligned}
& W_{1}=A(\bar{c}+\bar{d}), \\
& W_{2}=C(a+b)
\end{aligned}
$$

e

$$
W_{3}=C(c+d) \text {. }
$$

Lema 4.1.1. Com a construção acima, $p \in M$ é uma $H$-singularidade transversal se, e somente se,

$$
(a+b) \cdot(\bar{c}+\bar{d}) \neq 0 \text {. }
$$

Prova. A condição de transversalidade é dada por $N L \neq 0$, à qual é equivalente a (4.1.6). De fato, como estamos assumindo, por hipótese, que a curvatura normal no ponto $(0,0)$ é diferente de zero, e esta se escreve como 


$$
k_{N}(0,0)=A C \neq 0 \text {, }
$$

a afirmação acima é imediata.

Da análise que fizemos acima, resulta o seguinte teorema.

Teorema 4.1.2. Consideremos uma $H$-singularidade transversal onde $H(p)=0$ e $k_{N}(p) \neq 0$. Então temos:

i) $\mathrm{Se}$

$$
\frac{W_{2}}{W_{1}}>\frac{1}{2}\left[\left(\frac{W_{3}}{W_{1}}\right)^{2}+1\right]
$$

então a $H$-singularidade é do tipo $S_{1}$ (figura 4.1);

ii) $\mathrm{Se}$

$$
\frac{1}{2}\left[\left(\frac{W_{3}}{W_{1}}\right)^{2}+1\right]>\frac{W_{2}}{W_{1}}>0,2 W_{2} \neq W_{1}
$$

então a $H$-singularidade é do tipo $S_{2}$ (figura 4.1);

iii) $\quad \mathrm{Se}$

$$
\frac{W_{2}}{W_{1}}<0
$$

então a $H$-singularidade é do tipo $S_{3}$ (figura 4.1).

Prova. As singularidades do campo de vetores $X$ (4.6) sobre o eixo $p$ (reta projetiva), são dadas pelas raízes da seguinte equação cúbica (4.7) 


$$
\varphi(p)=T_{u}(0,0, p)+p T_{v}(0,0, p)=0 .
$$

Esta cúbica terá uma ou três raízes reais. Portanto, o campo de vetores $X$ tem:

(a) Uma única singularidade no ponto $(0,0, p=0)$. Neste caso o discriminante da equação quadrática é negativo. A singularidade $(0,0,0)$ do campo $X$ é uma sela, se, e somente se, (4.1.7) é verificado;

(b) Três singularidades nos pontos $(0,0,0),\left(0,0, p_{1}\right)$ e $\left(0,0, p_{2}\right)$, onde $0, p_{1}$ e $p_{2}$ são as raízes de $\varphi$. Neste caso o discriminante da equação quadrática é positivo. A singularidade $(0,0,0)$ é um nó e as singularidades $\left(0,0, p_{1}\right)$ e $\left(0,0, p_{2}\right)$ são duas selas, se, e somente se (4.1.8) é verificado;

(c) Três singularidades nos pontos $(0,0,0),\left(0,0, p_{1}\right)$ e $\left(0,0, p_{2}\right)$, onde $0, p_{1}$ e $p_{2}$ são as raízes de $\varphi$. Novamente neste caso o discriminante da equação quadrática é positivo. Estas três singularidades são três selas, se, e somente se, (4.1.9) é verificado.

As configurações locais das $H$-singularidades de tipo $S$, ilustradas na figura 4.1, são obtidas projetando-se, sobre o plano $u v$, as configurações das singularidades acima, conforme [GS1].

Observação 4.1.3. Convém, neste ponto, destacar a importância do sinal da curvatura normal. Por exemplo, suponhamos que a curvatura normal em $(0,0)$ seja positiva. Pelo teorema 3.1.3 sabemos que, neste caso, os sinais \pm do índice do campo $H$ na singularidade $(0,0), I(H,(0,0))$, e do índice da $H$-singularidade $(0,0)$, $i(0,0)$, concordam. Se $I(H,(0,0))=-1$ segue que $(0,0)$ é uma $H$-singularidade de tipo $S_{3}$, pois esta é a única com índice negativo. 


\subsection{O Caso dos Pontos de Inflexão}

Suponhamos que $p \in M$ seja uma $H$-singularidade, à qual é um ponto de inflexão com $H(p) \neq 0$. Uma vez que, neste caso, a elipse de curvatura é um segmento de reta radial podemos escolher apropriadamente uma rotação no plano normal de modo a escrever $r_{20}=r_{02}=r_{11}=0$. Agora, escolhendo apropriadamente uma rotação no plano $u v$ é possível escrever $\bar{b}=\bar{a}$. Assim, (4.1) pode ser escrito como

$$
\begin{gathered}
S(u, v)=\frac{A}{2}\left(u^{2}+v^{2}\right)+B u v+O(3) \\
R(u, v)=\frac{\bar{a}}{6} u^{3}+\frac{\bar{d}}{2} u^{2} v+\frac{\bar{a}}{2} u v^{2}+\frac{\bar{c}}{6} v^{3}+O(4) .
\end{gathered}
$$

Isto implica que (4.3) tem a forma

$$
\begin{gathered}
J=0, K=0, L=A(\bar{d}-\bar{c}), \\
M=0, N=2(\bar{a} B-A \bar{d}) \text { e } P=B(\bar{c}+\bar{d})-2 A \bar{a} .
\end{gathered}
$$

Definimos

$$
\begin{aligned}
& \overline{W_{1}}=A(\bar{d}-\bar{c}), \\
& \overline{W_{2}}=\bar{a} B-A \bar{d}
\end{aligned}
$$

$\mathrm{e}$

$$
\overline{W_{3}}=B(\bar{c}+\bar{d})-2 A \bar{a}
$$

Lema 4.2.1. Com as construções acima, $p \in M$ é uma $H$-singularidade transversal se, e somente se,

$$
\overline{W_{1}} \overline{W_{2}} \neq 0
$$


Prova. A condição de transversalidade, neste caso, é dada por $N L \neq 0$, à qual é equivalente a (4.2.6).

De modo análogo ao teorema 4.1.2 temos o seguinte teorema.

Teorema 4.2.2. Consideremos uma $H$-singularidade, à qual é um ponto de inflexão e $H(p) \neq 0$. Então, temos:

i) $\mathrm{Se}$

$$
\frac{\overline{W_{2}}}{\overline{W_{1}}}>\frac{1}{4}\left[\left(\frac{\overline{W_{3}}}{\overline{W_{1}}}\right)^{2}+1\right] \text {, }
$$

então a $H$-singularidade é do tipo $S_{1}$ (figura 4.1);

ii) $\mathrm{Se}$

$$
\frac{1}{4}\left[\left(\frac{\bar{W}_{3}}{\overline{W_{1}}}\right)^{2}+1\right]>\frac{\overline{W_{2}}}{\overline{W_{1}}}>0,4 \bar{W}_{2} \neq \bar{W}_{1}
$$

então a $H$-singularidade é do tipo $S_{2}$ (figura 4.1);

iii) $\quad \mathrm{Se}$

$$
\frac{\overline{W_{2}}}{\overline{W_{1}}}<0
$$

então a $H$-singularidade é do tipo $S_{3}$ (figura 4.1). 


\section{Capítulo 5. Linhas de Curvatura Direcional Média Periódicas}

O objetivo deste capítulo é estabelecer uma fórmula para a primeira derivada da aplicação de retorno (Poincaré) de uma linha de curvatura direcional média periódica em termos de invariantes geométricos da imersão.

Uma linha de curvatura direcional média periódica $\gamma$ da folheação $f_{H}(\alpha)$ (respectivamente $\left.F_{H}(\alpha)\right)$ é chamada de ciclo de curvatura direcional média mínimo (respectivamente máximo) de $\alpha$.

Denotemos por $\pi=\pi_{\gamma}$ a aplicação de (Poincaré) primeiro retorno definida pelas linhas de curvatura direcional média da folheação à qual $\gamma$ pertence, sobre um segmento de linha de curvatura direcional média da folheação ortogonal através de 0 em $\gamma$. Um ciclo de curvatura direcional média é chamado hiperbólico se a primeira derivada da aplicação de retorno for diferente de um, isto é, $\pi^{\prime}(0) \neq 1$. Neste caso, o ciclo de curvatura direcional média $\gamma$ atrai $\left(\pi^{\prime}(0)<1\right)$ as linhas de curvatura direcional média próximas ou as repele $\left(\pi^{\prime}(0)>1\right)$.

Seja $\gamma: I \rightarrow M^{2}$ um ciclo de curvatura direcional média da folheação mínima $f_{H}(\alpha)$, parametrizado pelo comprimento de arco $s$ e de período $L$. 
Tomemos $\left\{T_{1}(s)=\gamma^{\prime}(s), T_{2}(s)\right\}$ um referencial ortonormal de $T_{\gamma(s)} M$, notando que $T_{2}(s)$ é tangente à linha de curvatura direcional média ortogonal a $\gamma$, e $\left\{N_{1}(s), N_{2}(s)\right\} \quad$ um referencial ortonormal de $N_{\gamma(s)} M$, de modo que $\left\{T_{1}(s), T_{2}(s), N_{1}(s), N_{2}(s)\right\}$ seja um referencial positivo de $R^{4}$. Escolhemos o referencial $\left\{N_{1}(s), N_{2}(s)\right\}$ com as seguintes propriedades

$$
\eta\left(\gamma(s), T_{1}(s)\right)=a(s) H_{1}(s) N_{1}(s)
$$

$\mathrm{e}$

$$
\eta\left(\gamma(s), T_{2}(s)\right)=b(s) H_{1}(s) N_{1}(s) .
$$

Isto é obtido fazendo uma rotação em $N_{\gamma(s)} M$ de modo que o campo de vetores $N_{1}(s)$ esteja na direção do campo de vetores $H$. Isto é sempre possível, pois $H=H(s) \neq 0$, uma vez que $\gamma$ é uma linha de curvatura direcional média. Decorre desta observação que as funções $a$ e $b$ acima estão relacionadas por uma relação da forma $a+b \equiv 2$.

Podemos construir o referencial de Darboux associado a $\gamma$, da seguinte maneira:

$$
\begin{gathered}
T_{1}^{\prime}(s)=k_{g}(s) T_{2}(s)+\eta\left(\gamma(s), T_{1}(s)\right)=k_{g}(s) T_{2}(s)+a(s) H_{1}(s) N_{1}(s) ; \\
T_{2}^{\prime}(s)=-k_{g}(s) T_{1}(s)+\tau_{g}(s)=-k_{g}(s) T_{1}(s)+\tau_{g, 1}(s) N_{1}(s)+\tau_{g, 2}(s) N_{2}(s) ; \\
N_{1}^{\prime}(s)=-a(s) H_{1}(s) T_{1}(s)-\tau_{g, 1}(s) T_{2}(s)+\tau_{n}(s) N_{2}(s) ; \\
N_{2}^{\prime}(s)=-\tau_{g, 2}(s) T_{2}(s)-\tau_{n}(s) N_{1}(s) .
\end{gathered}
$$

Aqui $H=H_{1} N_{1}$ é o vetor curvatura média,

$$
\tau_{g}=\tau_{g, 1} N_{1}+\tau_{g, 2} N_{2}
$$


é o vetor torção geodésica e

$$
\tau_{n}=\left\langle N_{1}^{\prime}, N_{2}\right\rangle
$$

é a torção normal do referencial $\left\{N_{1}, N_{2}\right\}$.

No ponto $\gamma(s)$, a interseção da superfície $M$ com o hiperplano gerado por $\left\{T_{2}(s), N_{1}(s), N_{2}(s)\right\}$ é a curva $\Gamma_{s}$, tangente a $T_{2}(s)$ em $\gamma(s)$. Esta curva $\Gamma_{s}$ pode ser parametrizada por

$$
\Gamma_{s}(t)=\gamma(s)+t T_{2}(s)+V_{1}(s, t) N_{1}(s)+V_{2}(s, t) N_{2}(s)
$$

e sua curvatura é dada por $\eta\left(\gamma(s), T_{2}(s)\right)$. Destas considerações temos

$$
\Gamma_{s}(0)=\gamma(s), \quad \Gamma_{s}^{\prime}(0)=T_{2}(s) \text { e } \Gamma_{s}^{\prime \prime}(0)=b(s) H_{1}(\gamma(s)) N_{1}(s), \forall s \in[0, L]
$$

Segue que as funções $V_{1}$ e $V_{2}$ satisfazem

$$
V_{1}(s, 0)=V_{2}(s, 0)=\left.\frac{\partial\left[V_{1}(s, t)\right]}{\partial t}\right|_{t=0}=\left.\frac{\partial\left[V_{2}(s, t)\right]}{\partial t}\right|_{t=0}=\left.\frac{\partial^{2}\left[V_{2}(s, t)\right]}{\partial t^{2}}\right|_{t=0}=0
$$

e

$$
\left.\frac{\partial^{2}\left[V_{\mathrm{I}}(s, t)\right]}{\partial t^{2}}\right|_{t=0}=b(s) H_{1}(\gamma(s)), \forall s \in[0, L]
$$

Deste modo temos as seguintes expressões

e

$$
\begin{gathered}
V_{1}(s, t)=\frac{b(s) H_{1}(s)}{2} t^{2}+\bar{A}(s, t) \frac{t^{3}}{6}+O(4), \\
V_{2}(s, t)=\bar{B}(s, t) \frac{t^{3}}{6}+O(4)
\end{gathered}
$$

$$
\bar{A}(s)=\bar{A}(s, 0) \text { e } \bar{B}(s)=\bar{B}(s, 0) .
$$


Lema 5.1. Seja $\gamma$ um ciclo de curvatura direcional média da folheação $f_{H}(\alpha)$, parametrizado pelo comprimento de arco $s$ e de período $L$. Tomemos o referencial ortonormal positivo de Darboux $\left\{T_{1}, T_{2}, N_{1}, N_{2}\right\}$ ao longo de $\gamma$. Então a expressão (5.1) define um carta local de $M, L$ periódica em $s$, de classe $C^{k-5}$ em uma vizinhança de $\gamma$.

$$
\begin{aligned}
\alpha(s, t)=\gamma(s)+t T_{2}(s)+\left[\frac{b(s) H_{1}(s)}{2}\right. & \left.t^{2}+\bar{A}(s, t) \frac{t^{3}}{6}+O(4)\right] N_{1}(s)+ \\
+ & {\left[\bar{B}(s, t) \frac{t^{3}}{6}+O(4)\right] N_{2}(s), }
\end{aligned}
$$

Prova. O lema segue a partir do Teorema da Função Inversa e da aplicação

$$
\alpha\left(s, t, v_{1}, v_{2}\right)=\gamma(s)+t T_{2}(s)+v_{1} N_{1}(s)+v_{2} N_{2}(s)
$$

à qual é de classe $C^{k-2}$ e define uma vizinhança tubular de $\gamma$. As funções $\bar{A}$ e $\bar{B}$ em (5.1) são de classe $C^{k-5}$.

De (5.1) e do referencial de Darboux, temos

$$
\begin{gathered}
E(s, 0)=G(s, 0)=1, F(s, 0)=0, \\
e_{1}(s, 0)=a(s) H_{1}(s), f_{1}(s, 0)=\tau_{g, 1}(s), \\
g_{1}(s, 0)=b(s) H_{1}(s), \\
e_{2}(s, 0)=g_{2}(s, 0)=0 \text { e } f_{2}(s, 0)=\tau_{g, 2}(s) .
\end{gathered}
$$

Notemos que ao longo de $\gamma(s)$ temos

$$
k_{N}(s)=k_{N}(s, 0)=\tau_{g, 2}(s)(a(s)-b(s)) H_{1}(s)
$$


e

$$
\Delta(s)=\Delta(s, 0)=-\left(\tau_{g, 2}(s)\right)^{2} a(s) b(s)\left(H_{1}(s)\right)^{2} .
$$

Como $H_{1}(s)=\|H(s)\| \neq 0, \forall s \in[0, L]$, e as funções $a$ e $b$ não são simultaneamente nulas, devemos ter $\tau_{g, 2}(s) \neq 0, \forall s \in[0, L]$.

Lema 5.2. Seja $\gamma$ um ciclo de curvatura direcional média e consideremos a carta $(s, t)$ obtida em (5.1). Então o referencial ortonormal $\left\{N_{1}, N_{2}\right\}$ do fibrado normal satisfaz as seguintes equações

$$
\begin{gathered}
\left(N_{1}\right)_{t}(s, 0)=-\tau_{g, 1}(s) T_{1}(s)-b(s) H_{1}(s) T_{2}(s)+a_{12}^{3}(s) N_{2}(s), \\
\left(N_{2}\right)_{t}(s, 0)=-\tau_{g, 2}(s) T_{1}(s)-a_{12}^{3}(s) N_{1}(s)
\end{gathered}
$$

onde

$$
a_{12}^{3}(s)=a_{12}^{3}(s, 0)=\left\langle\left(N_{1}\right)_{t}(s, 0), N_{2}(s)\right\rangle
$$

é a torção normal do referencial $\left\{N_{1}, N_{2}\right\}$, associado à linha de curvatura direcional média ortogonal a $\gamma$ no ponto $\gamma(s)$.

Prova. Na carta $(s, t)$ as seguintes equações, às quais fazem parte das equações de estrutura, são satisfeitas

$$
\begin{aligned}
& \left(N_{1}\right)_{t}(s, 0)=\left(\frac{g_{1} F-f_{1} G}{E G-F^{2}}\right)(s, 0) \alpha_{s}(s, 0)+\left(\frac{f_{1} F-g_{1} E}{E G-F^{2}}\right)(s, 0) \alpha_{t}(s, 0)+a_{12}^{3}(s) N_{2}(s) \\
& \left(N_{2}\right)_{t}(s, 0)=\left(\frac{g_{2} F-f_{2} G}{E G-F^{2}}\right)(s, 0) \alpha_{s}(s, 0)+\left(\frac{f_{2} F-g_{2} E}{E G-F^{2}}\right)(s, 0) \alpha_{t}(s, 0)-a_{12}^{3}(s) N_{1}(s) .
\end{aligned}
$$

e

Utilizando (5.2), o lema está provado. 
Vamos, a seguir, calcular as derivadas com relação à variável $t$ dos coeficientes da primeira e segunda formas fundamentais.

$$
\begin{gathered}
E_{t}(s, 0)=\left\langle\gamma_{s}, \gamma_{s}\right\rangle_{t}(s, 0)=2\left\langle T_{2}^{\prime}, T_{1}\right\rangle=-2 k_{g}(s), \\
F_{t}(s, 0)=\left\langle\gamma_{s}, \gamma_{t}\right\rangle_{t}(s, 0)=0, \\
G_{t}(s, 0)=\left\langle\gamma_{t}, \gamma_{t}\right\rangle_{t}(s, 0)=2\left\langle T_{2}, b H_{1} N_{1}\right\rangle=0, \\
\left(e_{1}\right)_{t}(s, 0)=\left\langle\gamma_{s s}, N_{1}\right\rangle_{t}(s, 0)=\left\langle T_{2}^{\prime \prime}, N_{1}\right\rangle+\left\langle T_{1}^{\prime},-b H_{1} T_{2}+a_{12}^{3} N_{2}\right\rangle= \\
=\tau_{g, 1}^{\prime}-\tau_{g, 2} \tau_{n}-k_{g} H_{1}(a+b), \\
\left(f_{1}\right)_{t}(s, 0)=\left\langle\gamma_{s t}, N_{1}\right\rangle_{t}(s, 0)=\left\langle\left(b H_{1}\right)^{\prime} N_{1}, N_{1}\right\rangle+\left\langle T_{2}^{\prime},-\tau_{g, 1} T_{1}-b H_{1} T_{2}+a_{12}^{3} N_{2}\right\rangle= \\
=\left(b H_{1}\right)^{\prime}+k_{g} \tau_{g, 1}+\tau_{g, 2} a_{12}^{3}, \\
\left(g_{1}\right)_{t}(s, 0)=\left\langle\gamma_{t t}, N_{1}\right\rangle_{t}(s, 0)=\left\langle\bar{A} N_{1}+\bar{B} N_{2}, N_{1}\right\rangle+\left\langle b H_{1} N_{1},-\tau_{g, 1} T_{1}-b H_{1} T_{2}+a_{12}^{3} N_{2}\right\rangle= \\
=\bar{A}(s), \\
\left(e_{2}\right)_{t}(s, 0)=\left\langle\gamma_{s s}, N_{2}\right\rangle_{t}(s, 0)=\left\langle T_{2}^{\prime \prime}, N_{2}\right\rangle+\left\langle T_{1}^{\prime},-\tau_{g, 2} T_{1}-a_{12}^{3} N_{1}\right\rangle=\tau_{g, 1} \tau_{n}+\tau_{g, 2}^{\prime}-a H_{1} a_{12}^{3}, \\
=\left\langle\gamma_{s t}, N_{2}\right\rangle_{t}(s, 0)= \\
=\tau_{g, 1} a_{12}^{3} \\
\left.\left.f_{1}\right)_{1}, N_{2}\right\rangle+\left\langle-k_{g} T_{1}+\tau_{g, 1} N_{1}+\tau_{g, 2} N_{2},-\tau_{g, 2} T_{1}-a_{12}^{3} N_{1}\right\rangle=
\end{gathered}
$$

$\mathrm{e}$

$$
\begin{aligned}
\left(g_{2}\right)_{t}(s, 0) & =\left\langle\gamma_{t t}, N_{2}\right\rangle_{t}(s, 0)=\left\langle\bar{A} N_{1}+\bar{B} N_{2}, N_{2}\right\rangle+\left\langle b H_{1} N_{1},-\tau_{g, 2} T_{1}-a_{12}^{3} N_{1}\right\rangle= \\
& =\bar{B}(s)-b H_{1} a_{12}^{3} .
\end{aligned}
$$


Lema 5.3. A função $\bar{B}$, introduzida em (5.1), é dada por

$$
\bar{B}(s)=2\left(H_{2}\right)_{t}(s, 0)+H_{1}(s) a_{12}^{3}(s)(a(s)+b(s))-\tau_{g, 1}(s) \tau_{n}(s)-\tau_{g, 2}^{\prime}(s) .
$$

Prova. Na carta $(s, t)$ temos

$$
H_{2}(s, t)=\left[\frac{E g_{2}-2 F f_{2}+G e_{2}}{2\left(E G-F^{2}\right)}\right](s, t) .
$$

Derivando a função $H_{2}$ com relação à variável $t$ e utilizando (5.2), (5.3) e (5.4), o lema está provado.

Proposição 5.4. Seja $\gamma$ um ciclo de curvatura direcional média da folheação $f_{H}(\alpha)$, parametrizado pelo comprimento de arco $s$ e de período $L$. Então, a derivada da aplicação de primeiro retorno é dada por

$$
\pi^{\prime}(0)=\exp \left[\frac{1}{2} \int_{0}^{L}\left[\frac{a(s)\left(\left(H_{2}\right)_{t}(s)+H_{1}(s) a_{12}^{3}(s)\right)-\tau_{g, 1}(s) \tau_{n}(s)}{\tau_{g, 2}(s)}\right] d s\right]
$$

Prova. A derivada da aplicação de Poincaré satisfaz a seguinte equação diferencial linear

$$
\frac{d}{d s}\left(\frac{d t}{d t_{0}}\right)=-\frac{1}{2 B} \frac{\partial A}{\partial t} \frac{d t}{d t_{0}}
$$

Portanto,

$$
\pi^{\prime}(0)=\exp \left[\int_{0}^{L}\left(-\frac{A_{t}(s, 0)}{2 B(s, 0)}\right) d s\right]
$$

onde as funções $A$ e $B$ são dadas em (2.2.3) e (2.2.4). Utilizando (5.2), (5.3) e (5.4) temos 


$$
2 B(s, 0)=-2 H_{1}(s) \tau_{g, 2}(s)(a(s)+b(s)),
$$

e

$$
\begin{aligned}
A_{t}(s, 0) & =H_{1}\left[a \bar{B}-b\left(\tau_{g, 2}^{\prime}+\tau_{g, 1} \tau_{n}\right)\right]= \\
& =H_{1}\left[a\left(2\left(H_{2}\right)_{t}+H_{1} a_{12}^{3}(a+b)\right)-\left(\tau_{g, 2}^{\prime}+\tau_{g, 1} \tau_{n}\right)(a+b)\right]
\end{aligned}
$$

Deste modo,

$$
\begin{aligned}
\frac{-A_{t}(s, 0)}{2 B(s, 0)} & =\frac{-H_{1}\left\lfloor a\left(2\left(H_{2}\right)_{t}+H_{1} a_{12}^{3}(a+b)\right)-\left(\tau_{g, 2}^{\prime}+\tau_{g, 1} \tau_{n}\right)(a+b)\right\rfloor}{-2 H_{1} \tau_{g, 2}(a+b)}= \\
& =\frac{a(s)\left(\left(H_{2}\right)_{t}(s)+H_{1}(s) a_{12}^{3}(s)\right)-\tau_{g, 1}(s) \tau_{n}(s)}{2 \tau_{g, 2}(s)}-\frac{\tau_{g, 2}^{\prime}(s)}{2 \tau_{g, 2}(s)} .
\end{aligned}
$$

Notemos que

$$
\int_{\alpha}^{L}\left[\frac{\tau_{g, 2}^{\prime}(s)}{2 \tau_{g, 2}(s)}\right] d s=0
$$

Deste modo, de (5.7), temos

$$
\pi^{\prime}(0)=\exp \left[\frac{1}{2} \int_{0}^{L}\left(\frac{a(s)\left(\left(H_{2}\right)_{t}(s)+H_{1}(s) a_{12}^{3}(s)\right)-\tau_{g, 1}(s) \tau_{n}(s)}{\tau_{g, 2}(s)}\right) d s\right] .
$$

Observação 5.5. Quando $\gamma$ é um ciclo de curvatura direcional médio da folheação $F_{H}(\alpha)$ temos

$$
\pi^{\prime}(0)=\exp \left[\frac{1}{2} \int_{0}^{L}\left[\frac{b(s)\left(\left(H_{2}\right)_{t}(s)+H_{1}(s) a_{12}^{3}(s)\right)-\tau_{g, 1}(s) \tau_{n}(s)}{\tau_{g, 2}(s)}\right] d s\right] .
$$

Proposição 5.6. Seja $\alpha: M \rightarrow R^{4}$ uma imersão de classe $C^{r}, r \geq 6$, e seja $\gamma$ um ciclo de curvatura direcional média da folheação $f_{H}(\alpha)$, parametrizado pelo 
comprimento de arco e de comprimento $L$. Tome a carta $(s, t)$ como no lema $5.1 \mathrm{e}$ considere a seguinte deformação

$$
\beta_{\varepsilon}(u, v)=\beta(\varepsilon, u, v)=\alpha(u, v)+\varepsilon\left[\frac{a(s) \tau_{g, 2}(s)}{6} t^{3}\right] \delta(t) N_{2}(s)
$$

onde $\delta=1$ numa vizinhança de $t=0$, com suporte pequeno e a função $a$ não identicamente nula. Então, $\gamma$ é um ciclo de curvatura direcional média de $\beta_{\varepsilon}$, para todo $\varepsilon \geq 0$ pequeno, e mais, $\gamma$ é um ciclo de curvatura direcional média hiperbólico para $\beta_{\varepsilon}, \varepsilon \neq 0$.

Prova. Cálculos com a deformação $\beta_{\varepsilon}$ nos fornece

$$
2\left(H_{2}\right)_{t}(s, 0)=\tau_{g, 1}(s) \tau_{n}(s)+\tau_{g, 2}^{\prime}(s)-2 H_{1}(s) a_{12}^{3}(s)+\bar{B}(s)+\varepsilon a(s) \tau_{g, 2}(s) .
$$

Portanto, assumindo que a função $a$ não seja identicamente nula, temos

$$
\frac{d}{d \varepsilon}\left[\ln \pi^{\prime}(0)\right]_{\varepsilon=0}=\frac{1}{2} \int_{0}^{L} \frac{(a(s))^{2} \tau_{g, 2}(s)}{2 \tau_{g, 2}(s)} d s=\frac{1}{4} \int_{0}^{L}(a(s))^{2} d s \neq 0 .
$$

Observação 5.7. A expressão (5.6) é a derivada da aplicação de transição para uma folheação direcional média (a qual neste ponto é apenas de classe $C^{1}$ ), ao longo de um arco de linha de curvatura direcional média. Podemos aproximar uma imersão de classe $C^{3}$ por uma de classe $C^{6}$. A correspondente aplicação de transição (agora de classe $C^{4}$ ), cuja derivada é dada por (5.6), converge na classe $C^{1}$ para a aplicação de transição original, mas que cuja expressão deve ser dada pela mesma integral, uma vez que para as funções envolvidas existem limites uniformes, de acordo com [GaS2]. 


\section{Capítulo 6. Um Caso Especial}

Neste capítulo vamos fixar nossa atenção em uma situação, que embora pareça bastante particular, por um lado, torna-se também bastante interessante, por outro, pois serve de ponte para uma analogia entre superfícies imersas nos espaços $R^{3}$ e $R^{4}$. Mais especificamente vamos estudar as imersões de superfícies em $R^{4}$ estando estas em $S^{3}$, via aplicação inversa da projeção estereográfica de uma superfície imersa em $R^{3}$. Depois faremos um estudo comparativo, do ponto de vista de alguns campos de direções e suas singularidades, entre estas imersões, ou seja, a imersão de uma superfície em $R^{3}$ e sua imagem em $R^{4}$, via aplicação inversa da projeção estereográfica. Apresentamos na terceira seção deste capítulo uma tabela deste estudo comparativo. Na última seção deste capítulo falaremos a respeito das superfícies imersas em $R^{4}$ estando na esfera $S^{3}$.

\subsection{A Projeção de uma Superfície Imersa em $R^{3}$ em $S^{3}$}

Seja $\phi: R^{3} \rightarrow S^{3}$ a aplicação (inversa da) projeção estereográfica, dada por

$$
\phi(x, y, z)=\frac{1}{1+w}(x, y, z, w)
$$

onde 


$$
w=\frac{1}{2}\left(x^{2}+y^{2}+z^{2}-1\right)
$$

Recordemos que $\phi$ é um difeomorfismo de $R^{3}$ em $S^{3} \backslash\{(0,0,0,1)\}$ e é conforme. Seja $\alpha: M^{2} \rightarrow R^{3}$ uma imersão de uma superfície regular e orientada $M$ em $R^{3}$. Assumamos que $(u, v)$ seja uma carta positiva de $M$ e que $\left\{\alpha_{u}, \alpha_{v}, N\right\}$ seja um referencial positivo de $R^{3}$, onde $N=\frac{\alpha_{u} \wedge \alpha_{v}}{\left\|\alpha_{u} \wedge \alpha_{v}\right\|}$ é o campo normal na orientação de $\alpha$. Seja $\bar{\alpha}=\phi \circ \alpha$ a projeção estereográfica de $M$ em $S^{3}$. Consideremos a imersão de $M$ em $R^{4}$ via $\bar{\alpha}$, onde $\left\{\bar{\alpha}_{u}, \bar{\alpha}_{v}, N_{1}, N_{2}\right\}$ é um referencial positivo de $R^{4}$, sendo $N_{1}=\frac{d \phi(N)}{\|d \phi(N)\|}$ e $N_{2}$ a normal unitária interior a $S^{3}$. Em outras palavras estamos admitindo que $N_{2} \equiv-\bar{\alpha}$.

Vamos calcular os coeficientes da primeira e segunda formas fundamentais de $\alpha$ e $\bar{\alpha}$ para posteriormente fazermos um estudo comparativo das duas imersões.

Notemos que $\bar{\alpha}$ tem a seguinte escrita

$$
\bar{\alpha}(u, v)=\phi(\alpha(u, v))=\frac{1}{1+\langle\alpha(u, v), \alpha(u, v)\rangle}(2 \alpha(u, v),\langle\alpha(u, v), \alpha(u, v)\rangle-1) .
$$

Recordemos que os coeficientes da primeira forma fundamental de $\alpha$ são

$$
E(u, v)=\left\langle\alpha_{u}(u, v), \alpha_{u}(u, v)\right\rangle, F(u, v)=\left\langle\alpha_{u}(u, v), \alpha_{v}(u, v)\right\rangle
$$

e

$$
G(u, v)=\left\langle\alpha_{v}(u, v), \alpha_{v}(u, v)\right\rangle
$$

Derivando (6.1.1) com relação a $u$ e com relação a $v$, obtemos 


$$
\bar{\alpha}_{u}(u, v)=\frac{1}{1+\langle\alpha, \alpha\rangle}\left(2 \alpha_{u}, 2\left\langle\alpha_{u}, \alpha\right\rangle\right)-\frac{2\left\langle\alpha_{u}, \alpha\right\rangle}{(1+\langle\alpha, \alpha\rangle)^{2}}(2 \alpha,\langle\alpha, \alpha\rangle-1)
$$

e

$$
\bar{\alpha}_{v}(u, v)=\frac{1}{1+\langle\alpha, \alpha\rangle}\left(2 \alpha_{v}, 2\left\langle\alpha_{v}, \alpha\right\rangle\right)-\frac{2\left\langle\alpha_{v}, \alpha\right\rangle}{(1+\langle\alpha, \alpha\rangle)^{2}}(2 \alpha,\langle\alpha, \alpha\rangle-1) .
$$

Deste modo podemos calcular os coeficientes da primeira forma fundamental de $\bar{\alpha}$, obtendo

$$
\begin{aligned}
& \bar{E}(u, v)=\left\langle\bar{\alpha}_{u}(u, v), \bar{\alpha}_{u}(u, v)\right\rangle=\frac{4}{(1+\langle\alpha(u, v), \alpha(u, v)\rangle)^{2}}\left\langle\alpha_{u}(u, v), \alpha_{u}(u, v)\right\rangle, \\
& \bar{F}(u, v)=\left\langle\bar{\alpha}_{u}(u, v), \bar{\alpha}_{v}(u, v)\right\rangle=\frac{4}{(1+\langle\alpha(u, v), \alpha(u, v)\rangle)^{2}}\left\langle\alpha_{u}(u, v), \alpha_{v}(u, v)\right\rangle
\end{aligned}
$$

e

$$
\bar{G}(u, v)=\left\langle\bar{\alpha}_{v}(u, v), \bar{\alpha}_{v}(u, v)\right\rangle=\frac{4}{(1+\langle\alpha(u, v), \alpha(u, v)\rangle)^{2}}\left\langle\alpha_{v}(u, v), \alpha_{v}(u, v)\right\rangle .
$$

Observemos que

$$
\frac{4}{(1+\langle\alpha(u, v), \alpha(u, v)\rangle)^{2}}=\frac{1}{(1+w)^{2}}
$$

Logo, podemos escrever (6.1.4), (6.1.5) e (6.1.6) como

$$
\begin{aligned}
& \bar{E}(u, v)=\frac{1}{(1+w)^{2}} E(u, v), \\
& \bar{F}(u, v)=\frac{1}{(1+w)^{2}} F(u, v)
\end{aligned}
$$

$\mathrm{e}$

$$
\bar{G}(u, v)=\frac{1}{(1+w)^{2}} G(u, v) \text {. }
$$


De posse dos coeficientes da primeira forma fundamental podemos calcular a norma do vetor normal $d \phi(N)$, recordando que o mesmo é utilizado para obtermos o vetor $N_{1}$, através de

$$
N_{1}=\frac{d \phi(N)}{\|d \phi(N)\|} .
$$

Assim,

$$
\|d \phi(N)\|=\left(\bar{E} \bar{G}-\bar{F}^{2}\right)^{1 / 2}=\frac{1}{(1+w)^{2}}\left(E G-F^{2}\right)^{1 / 2}=\frac{1}{(1+w)^{2}}\|N\| .
$$

Para o cálculo dos coeficientes da segunda forma fundamental de $\bar{\alpha}$, faremos a seguinte convenção

$$
\bar{e}_{1}(u, v)=\left\langle\bar{\alpha}_{u u}, N_{1}\right\rangle=\left\langle\bar{\alpha}_{u u}, \frac{d \phi(N)}{\|d \phi(N)\|}\right\rangle=\frac{(1+w)^{2}}{\|N\|} \operatorname{det}\left[\bar{\alpha}_{u}, \bar{\alpha}_{v}, \bar{\alpha}, \bar{\alpha}_{u u}\right]
$$

e, de modo análogo,

$$
\bar{f}_{1}(u, v)=\left\langle\bar{\alpha}_{u v}, N_{1}\right\rangle=\frac{(1+w)^{2}}{\|N\|} \operatorname{det}\left[\bar{\alpha}_{u}, \overline{\alpha_{v}}, \bar{\alpha}, \bar{\alpha}_{u v}\right]
$$

$\mathrm{e}$

$$
\bar{g}_{1}(u, v)=\left\langle\bar{\alpha}_{v v}, N_{1}\right\rangle=\frac{(1+w)^{2}}{\|N\|} \operatorname{det}\left[\bar{\alpha}_{u}, \overline{\alpha_{v}}, \bar{\alpha}, \bar{\alpha}_{v v}\right] .
$$

Passemos aos cálculos dos determinantes acima. Definamos

$$
\begin{gathered}
A=\frac{1}{1+\langle\alpha, \alpha\rangle}, \\
B=\frac{2\left\langle\alpha_{u}, \alpha\right\rangle}{(1+\langle\alpha, \alpha\rangle)^{2}},
\end{gathered}
$$




$$
\begin{gathered}
C=\frac{2\left\langle\alpha_{v}, \alpha\right\rangle}{(1+\langle\alpha, \alpha\rangle)^{2}} \\
D_{1}=\frac{2\left(\left\langle\alpha_{u u}, \alpha\right\rangle+\left\langle\alpha_{u}, \alpha_{u}\right\rangle\right)(1+\langle\alpha, \alpha\rangle)-8\left\langle\alpha_{u}, \alpha\right\rangle^{2}}{(1+\langle\alpha, \alpha\rangle)^{3}}, \\
D_{2}=\frac{2\left(\left\langle\alpha_{u v}, \alpha\right\rangle+\left\langle\alpha_{u}, \alpha_{v}\right\rangle\right)(1+\langle\alpha, \alpha\rangle)-8\left\langle\alpha_{u}, \alpha\right\rangle\left\langle\alpha_{v}, \alpha\right\rangle}{(1+\langle\alpha, \alpha\rangle)^{3}},
\end{gathered}
$$

$\mathrm{e}$

$$
D_{3}=\frac{2\left(\left\langle\alpha_{v v}, \alpha\right\rangle+\left\langle\alpha_{v}, \alpha_{v}\right\rangle\right)(1+\langle\alpha, \alpha\rangle)-8\left\langle\alpha_{v}, \alpha\right\rangle^{2}}{(1+\langle\alpha, \alpha\rangle)^{3}} .
$$

Derivando as expressões (6.1.2) e (6.1.3) com relação a $u$ e $v$, obtemos

$$
\begin{gathered}
\bar{\alpha}_{u u}=2 A\left(\alpha_{u u},\left\langle\alpha_{u u}, \alpha\right\rangle+\left\langle\alpha_{u}, \alpha_{u}\right\rangle\right)-4 B\left(\alpha_{u},\left\langle\alpha_{u}, \alpha\right\rangle\right)-D_{1}(2 \alpha,\langle\alpha, \alpha\rangle-1), \\
\bar{\alpha}_{u v}=2 A\left(\alpha_{u v},\left\langle\alpha_{u v}, \alpha\right\rangle+\left\langle\alpha_{u}, \alpha_{v}\right\rangle\right)-2 C\left(\alpha_{u},\left\langle\alpha_{u}, \alpha\right\rangle\right)- \\
-2 B\left(\alpha_{v},\left\langle\alpha_{v}, \alpha\right\rangle\right)-D_{2}(2 \alpha,\langle\alpha, \alpha\rangle-1) \\
\bar{\alpha}_{v v}=2 A\left(\alpha_{v v},\left\langle\alpha_{v v}, \alpha\right\rangle+\left\langle\alpha_{v}, \alpha_{v}\right\rangle\right)-4 C\left(\alpha_{v},\left\langle\alpha_{v}, \alpha\right\rangle\right)-D_{3}(2 \alpha,\langle\alpha, \alpha\rangle-1) .
\end{gathered}
$$

$\mathrm{e}$

Utilizando as expressões de (6.1.14) a (6.1.22) e propriedades do determinante encontramos

$$
\begin{aligned}
\operatorname{det}\left[\bar{\alpha}_{u}, \bar{\alpha}_{v}, \bar{\alpha}, \bar{\alpha}_{u u}\right] & =8 A^{4}\left\{2\left(\left\langle\alpha_{u u}, \alpha\right\rangle+\left\langle\alpha_{u}, \alpha_{u}\right\rangle\right) \operatorname{det}\left[\alpha, \alpha_{u}, \alpha_{v}\right]+\right. \\
& +2\left\langle\alpha_{u}, \alpha\right\rangle \operatorname{det}\left[\alpha, \alpha_{v}, \alpha_{u u}\right]-2\left\langle\alpha_{v}, \alpha\right\rangle \operatorname{det}\left[\alpha, \alpha_{u}, \alpha_{u u}\right]- \\
& \left.-(\langle\alpha, \alpha\rangle-1) \operatorname{det}\left[\alpha_{u}, \alpha_{v}, \alpha_{u u}\right]\right\},
\end{aligned}
$$




$$
\begin{aligned}
\operatorname{det}\left[\bar{\alpha}_{u}, \bar{\alpha}_{v}, \bar{\alpha}, \bar{\alpha}_{u v}\right] & =8 A^{4}\left\{2\left(\left\langle\alpha_{u v}, \alpha\right\rangle+\left\langle\alpha_{u}, \alpha_{v}\right\rangle\right) \operatorname{det}\left[\alpha, \alpha_{u}, \alpha_{v}\right]\right. \\
& +2\left\langle\alpha_{u}, \alpha\right\rangle \operatorname{det}\left[\alpha, \alpha_{v}, \alpha_{u v}\right]-2\left\langle\alpha_{v}, \alpha\right\rangle \operatorname{det}\left[\alpha, \alpha_{u}, \alpha_{u v}\right]- \\
& \left.-(\langle\alpha, \alpha\rangle-1) \operatorname{det}\left[\alpha_{u}, \alpha_{v}, \alpha_{u v}\right]\right\}
\end{aligned}
$$

$\mathrm{e}$

$$
\begin{aligned}
\operatorname{det}\left[\bar{\alpha}_{u}, \bar{\alpha}_{v}, \bar{\alpha}, \bar{\alpha}_{v v}\right] & =8 A^{4}\left\{2\left(\left\langle\alpha_{v v}, \alpha\right\rangle+\left\langle\alpha_{v}, \alpha_{v}\right\rangle\right) \operatorname{det}\left[\alpha, \alpha_{u}, \alpha_{v}\right]+\right. \\
& +2\left\langle\alpha_{u}, \alpha\right\rangle \operatorname{det}\left[\alpha, \alpha_{v}, \alpha_{v v}\right]-2\left\langle\alpha_{v}, \alpha\right\rangle \operatorname{det}\left[\alpha, \alpha_{u}, \alpha_{v v}\right]- \\
& \left.-(\langle\alpha, \alpha\rangle-1) \operatorname{det}\left[\alpha_{u}, \alpha_{v}, \alpha_{v v}\right]\right\}
\end{aligned}
$$

Consideremos as seguintes funções

$$
\begin{aligned}
& h_{1}(u, v)=\frac{\left\langle\alpha_{u}, \alpha\right\rangle \operatorname{det}\left[\alpha, \alpha_{v}, \alpha_{u u}\right]-\left\langle\alpha_{v}, \alpha\right\rangle \operatorname{det}\left[\alpha, \alpha_{u}, \alpha_{u u}\right]}{\left(E G-F^{2}\right)^{1 / 2}}, \\
& h_{2}(u, v)=\frac{\left\langle\alpha_{u}, \alpha\right\rangle \operatorname{det}\left[\alpha, \alpha_{v}, \alpha_{u v}\right]-\left\langle\alpha_{v}, \alpha\right\rangle \operatorname{det}\left[\alpha, \alpha_{u}, \alpha_{u v}\right]}{\left(E G-F^{2}\right)^{1 / 2}}, \\
& h_{3}(u, v)=\frac{\left\langle\alpha_{u}, \alpha\right\rangle \operatorname{det}\left[\alpha, \alpha_{v}, \alpha_{v v}\right]-\left\langle\alpha_{v}, \alpha\right\rangle \operatorname{det}\left[\alpha, \alpha_{u}, \alpha_{v v}\right]}{\left(E G-F^{2}\right)^{1 / 2}},
\end{aligned}
$$

bem como a função suporte de $\alpha$, dada por

$$
t(u, v)=\langle\alpha(u, v), N(u, v)\rangle .
$$

De (6.1.11) e de (6.1.23), utilizando (6.1.26) e (6.1.29), podemos escrever

$$
\bar{e}_{1}(u, v)=(1+w)^{-2}\left[\left(\left\langle\alpha_{u u}, \alpha\right\rangle+E\right) t+h_{1}-w e(u, v)\right] .
$$


De (6.1.12) e de (6.1.24), utilizando (6.1.27) e (6.1.29), podemos escrever

$$
\bar{f}_{1}(u, v)=(1+w)^{-2}\left[\left(\left\langle\alpha_{u v}, \alpha\right\rangle+F\right) t+h_{2}-w f(u, v)\right] .
$$

De (6.1.13) e de (6.1.25), utilizando (6.1.28) e (6.1.29), podemos escrever

$$
\bar{g}_{1}(u, v)=(1+w)^{-2}\left[\left(\left\langle\alpha_{v v}, \alpha\right\rangle+G\right) t+h_{3}-w g(u, v)\right] .
$$

Agora, cálculos diretos permite-nos escrever

$$
\begin{gathered}
\left\langle\alpha_{u u}(u, v), \alpha(u, v)\right\rangle t(u, v)+h_{1}(u, v)=\langle\alpha(u, v), \alpha(u, v)\rangle e(u, v)=(2 w+1) e(u, v), \\
\left\langle\alpha_{u v}(u, v), \alpha(u, v)\right\rangle t(u, v)+h_{2}(u, v)=\langle\alpha(u, v), \alpha(u, v)\rangle f(u, v)=(2 w+1) f(u, v)
\end{gathered}
$$

os quais substituídos nas expressões (6.1.30), (6.1.31) e (6.1.32), respectivamente, fornecem-nos

$$
\begin{aligned}
& \bar{e}_{1}(u, v)=(1+w)^{-2}[(1+w) e(u, v)+E(u, v) t(u, v)] \\
& \bar{f}_{1}(u, v)=(1+w)^{-2}[(1+w) f(u, v)+F(u, v) t(u, v)] \\
& \bar{g}_{1}(u, v)=(1+w)^{-2}[(1+w) g(u, v)+G(u, v) t(u, v)] .
\end{aligned}
$$

Falta-nos calcular o segundo conjunto de coeficientes da segunda forma fundamental. Assim, temos

$$
\begin{aligned}
& \bar{e}_{2}(u, v)=\left\langle\bar{\alpha}_{u u}(u, v), N_{2}(u, v)\right\rangle=-\left\langle\bar{\alpha}_{u u}(u, v), \bar{\alpha}(u, v)\right\rangle, \\
& \bar{f}_{2}(u, v)=\left\langle\bar{\alpha}_{u v}(u, v), N_{2}(u, v)\right\rangle=-\left\langle\bar{\alpha}_{u v}(u, v), \bar{\alpha}(u, v)\right\rangle
\end{aligned}
$$

e

$$
\bar{g}_{2}(u, v)=\left\langle\bar{\alpha}_{v v}(u, v), N_{2}(u, v)\right\rangle=-\left\langle\bar{\alpha}_{v v}(u, v), \bar{\alpha}(u, v)\right\rangle .
$$


Utilizando as expressões (6.1.1), (6.1.20) e (6.1.36), podemos calcular

$$
\bar{e}_{2}(u, v)=(1+w)^{-2} E(u, v)=\bar{E}(u, v) .
$$

Utilizando as expressões (6.1.1), (6.1.21) e (6.1.37), podemos calcular

$$
\bar{f}_{2}(u, v)=(1+w)^{-2} F(u, v)=\bar{F}(u, v) .
$$

Utilizando as expressões (6.1.1), (6.1.22) e (6.1.38), podemos calcular

$$
\bar{g}_{2}(u, v)=(1+w)^{-2} G(u, v)=\bar{G}(u, v) .
$$

De posse dos coeficientes da primeira e segunda formas fundamentais de $\bar{\alpha}$ podemos calcular o vetor curvatura média $\bar{H}$, o qual de (2.1.1), tem suas componentes dadas por

$$
\begin{aligned}
\bar{H}_{1}(u, v) & =\frac{\bar{e}_{1} \bar{G}-2 \bar{f}_{1} \bar{F}+\bar{g}_{1} \bar{E}}{2\left(\bar{E} \bar{G}-\bar{F}^{2}\right)}= \\
& =(1+w) \frac{e G-2 f F+g E}{2\left(E G-F^{2}\right)}+t \frac{2\left(E G-F^{2}\right)}{2\left(E G-F^{2}\right)}= \\
& =(1+w) H(u, v)+t(u, v)
\end{aligned}
$$

e

$$
\begin{aligned}
\bar{H}_{2}(u, v) & =\frac{\bar{e}_{2} \bar{G}-2 \bar{f}_{2} \bar{F}+\bar{g}_{2} \bar{E}}{2\left(\bar{E} \bar{G}-\bar{F}^{2}\right)}=\frac{\bar{E} \bar{G}-2 \overline{F F}+\bar{G} \bar{E}}{2\left(\bar{E} \bar{G}-\bar{F}^{2}\right)}= \\
& =\frac{2\left(\bar{E} \bar{G}-\bar{F}^{2}\right)}{2\left(\bar{E} \bar{G}-\bar{F}^{2}\right)}=1, \forall(u, v) .
\end{aligned}
$$

De posse da expressão (6.1.43) podemos afirmar: 
A elipse de curvatura $\varepsilon_{\bar{\alpha}}(p)$ está degenerada em um segmento de reta, sobre a reta suporte $N_{2}=1$, para todo $p \in M$. E ainda, como $\bar{H}(p) \neq 0, \forall p \in M$, as possíveis $\bar{H}$-singularidades só poderão ocorrer em pontos de inflexão, onde a elipse de curvatura se torna um segmento de reta radial, ou seja, a elipse de curvatura se torna um ponto, neste caso. Veja figura 6.1.1.

Com a finalidade de simplificar alguns cálculos voltemos a tomar uma carta $(u, v)$ na qual a primeira forma fundamental de $\alpha$ está diagonalizada com $E=G=1$ e $F=0$.

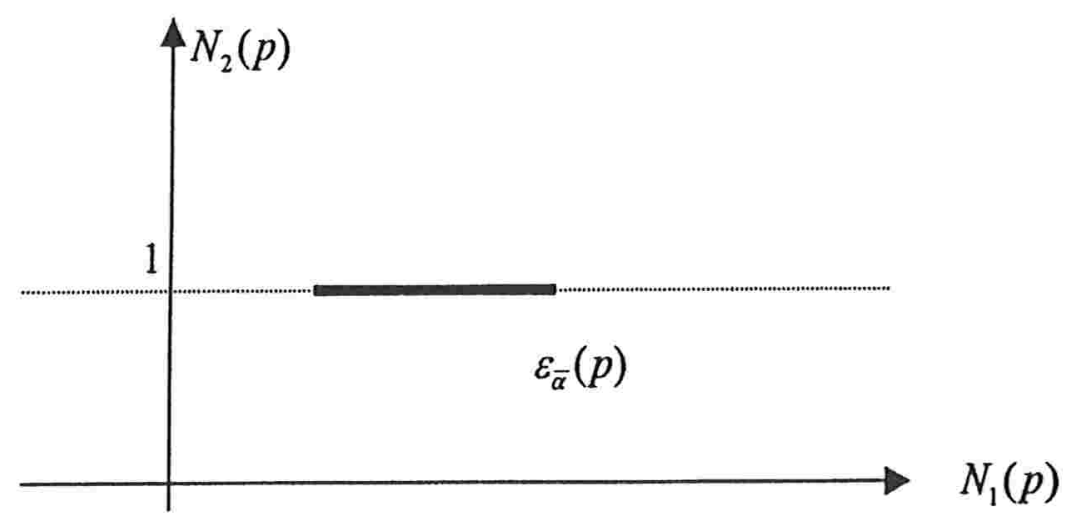

Figura 6.1.1. A elipse de curvatura de $\varepsilon_{\bar{\alpha}}(p)$.

Nesta carta os coeficientes da primeira e segunda formas fundamentais de $\bar{\alpha}$ são

$$
\begin{gathered}
\bar{E}(u, v)=\bar{G}(u, v)=\bar{e}_{2}(u, v)=\bar{g}_{2}(u, v)=(1+w)^{-2}, \\
\bar{F}(u, v)=0,
\end{gathered}
$$




$$
\begin{gathered}
\bar{e}_{1}(u, v)=(1+w)^{-2}[(1+w) e(u, v)+t(u, v)], \\
\bar{f}_{1}(u, v)=(1+w)^{-1} f(u, v)
\end{gathered}
$$

$\mathrm{e}$

$$
\bar{g}_{1}(u, v)=(1+w)^{-2}[(1+w) g(u, v)+t(u, v)]
$$

onde os símbolos sem a barra referem-se à imersão $\alpha$. Utilizando (2.1.2) é imediato a verificação de que a curvatura normal de $\bar{\alpha}$ é nula em todo ponto, como queríamos, uma vez que ela está associada à área da elipse de curvatura, que neste caso é nula em todos os pontos. Agora, de (2.1.3), a resultante $\Delta$ é dada por

$$
\Delta(u, v)=-(1+w)^{-6}\left[(f(u, v))^{2}+\left(\frac{e(u, v)-g(u, v)}{2}\right)^{2}\right] .
$$

Deste modo a resultante $\Delta$ anula-se nos pontos $(u, v)$ onde $f(u, v)=0$ e $e(u, v)-g(u, v)=0$, isto é, nos pontos umbílicos de $\alpha$. Temos demonstrado o seguinte teorema.

Teorema 6.1.1. Com as construções acima, um ponto $(u, v)$ é uma $\bar{H}$ singularidade de $\bar{\alpha}$, se e somente se, for um ponto umbílico de $\alpha$.

\subsection{Estudo dos Campos de Direções}

Na seção 2.1 do capítulo 2 fizemos referência a respeito de dois outros campos de direções sobre superfícies em $R^{4}$, os campos de direções assintóticas e os campos de direções de curvatura axial (veja figuras 2.1.2 e 2.1.3). De [GaS1], podemos escrever a equação diferencial dos campos de direções de curvatura axial como

$$
\operatorname{Jac}\left(\|\eta-H\|^{2}, I\right)=0
$$


onde $\eta, H$ e $I$ são respectivamente o vetor curvatura normal, o vetor curvatura média e a primeira forma fundamental da imersão $\alpha: M^{2} \rightarrow R^{4}$. Desenvolvendo (6.2.1) podemos escrever a seguinte equação diferencial quártica

$$
a_{4} d v^{4}+a_{3} d v^{3} d u+a_{2} d v^{2} d u^{2}+a_{1} d v d u^{3}+a_{0} d u^{4}=0
$$

onde $a_{4}, a_{3}, a_{2}, a_{1}$ e $a_{0}$ são funções dos coeficientes da primeira e segunda formas fundamentais e $(u, v)$ é uma carta local de $M$. Quando a imersão $\alpha$ está, em particular, contida em um hiperplano de $R^{4}$ a equação quártica (6.2.1) pode ser fatorada num produto de duas equações quadráticas, a saber, a equação quadrática das linhas de curvatura principal e a equação quadrática das linhas de curvatura média. Para o estudo da equação quadrática das linhas de curvatura principal recomendamos o texto [GS1] e para o estudo da equação quadrática das linhas de curvatura média recomendamos o artigo [GaS2].

Vejamos um pouco da parte geométrica intuitiva a respeito da elipse de curvatura de uma imersão $\bar{\alpha}: M^{2} \rightarrow R^{4}$, via projeção estereográfica de uma imersão $\alpha: M^{2} \rightarrow R^{3}$, como na seção anterior. Como vimos (figura 6.1.1), a elipse de curvatura $\varepsilon_{\bar{\alpha}}(p)$ é um segmento de reta, sobre a reta suporte $N_{2}=1$, para todo ponto $p$. Agora, por definição, o campo de direções assintóticas é o campo induzido no espaço tangente de $\bar{\alpha}$ pelos pontos onde o vetor curvatura normal tangencia a elipse de curvatura (veja figura 2.1.2) e, por definição, o campo de direções de curvatura direcional média é o campo induzido no espaço tangente de $\bar{\alpha}$ pelos pontos onde a reta gerada pelo vetor curvatura normal intercepta a elipse de curvatura. Ocorre que, neste caso, os pontos descritos acima coincidem com os vértices da elipse de curvatura $\varepsilon_{\bar{\alpha}}(p)$, para todo ponto $p$, exceto nos casos onde a elipse de curvatura se torna um ponto, ou seja, um segmento de 
reta radial, ou seja, um ponto de inflexão. Mas, os vértices da elipse de curvatura determinam os pontos que induzem no espaço tangente de $\bar{\alpha}$ o campo de direções de curvatura axial. Em outras palavras, também neste caso, a exemplo do caso em que a imersão de $M$ está contida em um hiperplano de $R^{4}$, o campo de direções de curvatura axial pode ser fatorado num produto de campos, o de direções assintóticas e o de direções de curvatura direcional média. Veja figura 6.2.1.

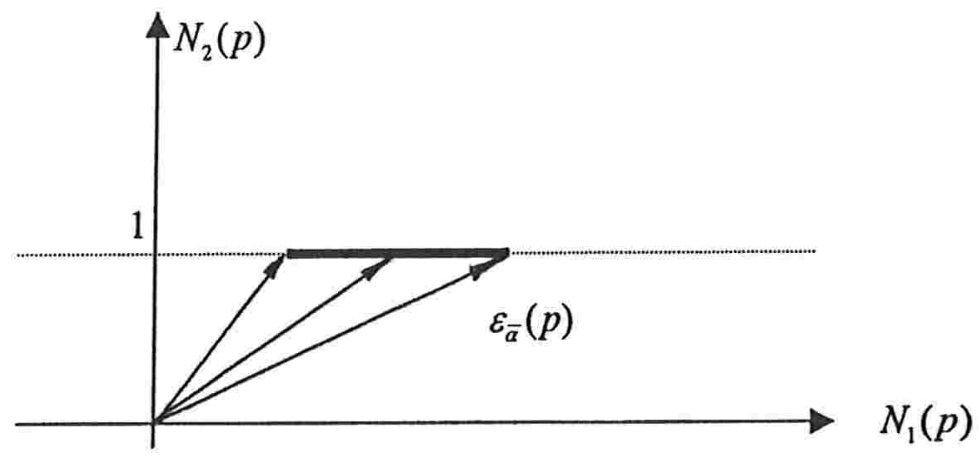

Figura 6.2.1. Os vértices da elipse de curvatura.

Teorema 6.2.1. Suponha que $\alpha: M^{2} \rightarrow R^{3}$ seja uma imersão de uma superfície regular e orientada $M$ em $R^{3}$ e $\bar{\alpha}: M^{2} \rightarrow R^{4}$ a imersão dada pela composição de $\alpha$ com a projeção estereográfica, como na seção anterior. Então, a equação diferencial quártica (6.2.1) é o produto da equação diferencial quadrática das linhas de curvatura direcional média (2.2.5) e da equação diferencial quadrática das linhas assintóticas, isto é, a equação (6.2.1) pode ser escrita como

$$
\operatorname{Jac}\left\{\operatorname{Jac}\left(I I_{1}, I\right), I\right\} . J a c\left(I I_{1}, I\right)=0 .
$$


Prova. É imediata. Tome a expressão (6.2.2) e a escreva com os coeficientes encontrados na seção anterior. Desenvolva o produto e compare o resultado com a equação quártica (6.2.1).

Utilizando as construções acima podemos observar que:

i) As linhas de curvatura principal de $\alpha$ são aplicadas, via projeção estereográfica, nas linhas assintóticas de $\bar{\alpha}$, as quais neste caso são ortogonais, fato este que pode ser facilmente verificado uma vez que a projeção estereográfica é conforme, preservando ângulos entre as curvas, ou ainda diretamente da elipse de curvatura, uma vez que neste caso os pontos de tangência do vetor curvatura normal com a elipse de curvatura são simétricos com relação ao centro da elipse, para todo ponto $p$ não pertencente ao conjunto das $\bar{H}$-singularidades;

ii) As linhas de curvatura média de $\alpha$ são aplicadas nas linhas de curvatura direcional média de $\bar{\alpha}$;

iii) As singularidades das linhas de curvatura principal e das linhas de curvatura média de $\alpha$, os pontos umbílicos, são aplicadas nas singularidades das linhas assintóticas e das linhas de curvatura direcional média de $\bar{\alpha}$, as $\bar{H}$-singularidades.

Observação 6.2.2. Baseados no teorema 6.2.1 podemos dar uma nova escrita para a equação diferencial das linhas assintóticas de uma imersão $\alpha: M^{2} \rightarrow R^{4}$, da qual a escrita que lá aparece é um caso particular, a saber,

$$
\operatorname{Jac}\left(I I_{1}, I_{2}\right)=0 \text {. }
$$


Esta escrita para a equação diferencial das linhas assintóticas é válida para qualquer imersão $\alpha: M^{2} \rightarrow R^{4}$. Utilizando os coeficientes da primeira e segunda formas fundamentais desta imersão podemos escrevê-la da seguinte maneira

$$
T_{3}(u, v) d u^{2}+T_{1}(u, v) d u d v+T_{2}(u, v) d v^{2}=0,
$$

onde as funções $T_{1}, T_{2}$ e $T_{3}$ foram definidas em (3.2.1) e $(u, v)$ é uma carta positiva de $M$. Como vimos na observação 3.2.4 as singularidades destes campos, os pontos de inflexão, são caracterizadas pelos pontos onde duas das funções acima se anulam.

\subsection{Quadro Comparativo das Imersões}

Apresentamos na página seguinte uma tabela comparando elementos das imersões $\alpha$ e $\bar{\alpha}$. Para a sua construção levamos em consideração os resultados obtidos nas seções anteriores. Para um melhor entendimento dos elementos que aparecem nesta tabela, bem como uma idéia de como eles foram obtidos, é interessante, pelo menos a visualização da figura 6.2.1, que trata dos vértices da elipse de curvatura para o caso considerado.

\subsection{Superfícies na Esfera $S^{3}$}

Seja $\alpha: M^{2} \rightarrow S^{3}$ uma imersão de classe $C^{r}, r \geq 4$, de uma superfície regular e orientada $M$ em $S^{3}$. Consideremos ainda a inclusão natural de $S^{3}$ em $R^{4}$. Assumamos que $(u, v)$ seja uma carta positiva de $M$ e que $\left\{\alpha_{u}, \alpha_{v}, N_{1}, N_{2}\right\}$ seja um referencial positivo de $R^{4}$, sendo $\left\{N_{1}, N_{2}\right\}$ um referencial de campo de vetores 
ortonormais a $\alpha$, onde $N_{1}(p) \in T_{p} S^{3}$ e $N_{2}(p)$ é a normal interior de $S^{3}, \forall p \in M$. Deste modo, temos $N_{2} \equiv-\alpha$.

\begin{tabular}{|c|c|c|c|}
\hline Imersão & $\alpha$ & $\bar{\alpha}$ & \\
\hline \multirow{3}{*}{$\begin{array}{l}\text { Primeira Forma } \\
\text { Fundamental }\end{array}$} & $E$ & \multicolumn{2}{|l|}{$\bar{E}=(1+w)^{-2} E$} \\
\hline & $F$ & \multicolumn{2}{|l|}{$\bar{F}=(1+w)^{-2} F$} \\
\hline & $G$ & \multicolumn{2}{|l|}{$\bar{G}=(1+w)^{-2} G$} \\
\hline \multirow{3}{*}{$\begin{array}{l}\text { Segunda Forma } \\
\text { Fundamental }\end{array}$} & $e$ & $\bar{e}_{1}=(1+w)^{-2}[(1+w) e+E t]$ & $\bar{e}_{2}=\bar{E}$ \\
\hline & $f$ & $\bar{f}_{1}=(1+w)^{-2}[(1+w) f+F t]$ & $\bar{f}_{2}=\bar{F}$ \\
\hline & $g$ & $\bar{g}_{1}=(1+w)^{-2}[(1+w) g+G t]$ & $\bar{g}_{2}=\bar{G}$ \\
\hline \multirow{5}{*}{$\begin{array}{c}\text { Campos de } \\
\text { Linhas e } \\
\text { Singularidades }\end{array}$} & Pontos Umbílicos & \multicolumn{2}{|l|}{ Pontos de Inflexão } \\
\hline & $\begin{array}{c}\text { Linhas de Curvatura } \\
\text { Principal }\end{array}$ & \multicolumn{2}{|l|}{ Linhas Assintóticas } \\
\hline & Linhas de Curvatura & \multirow{2}{*}{\multicolumn{2}{|c|}{$\begin{array}{c}\text { Linhas de Curvatura Direcional } \\
\text { Média }\end{array}$}} \\
\hline & Média & & \\
\hline & $\begin{array}{c}\text { Umbílicos } \\
\text { Darbouxianos } D_{i}\end{array}$ & \multicolumn{2}{|c|}{ Pontos de Inflexão do tipo $S_{i}$} \\
\hline
\end{tabular}

Tabela 6.3.1

Em tal carta $(u, v)$ valem

$$
e_{2}=\left\langle\alpha_{u u}, N_{2}\right\rangle=\left\langle\alpha_{u}, \alpha_{u}\right\rangle=E,
$$




$$
\begin{aligned}
& f_{2}=\left\langle\alpha_{u v}, N_{2}\right\rangle=\left\langle\alpha_{u}, \alpha_{v}\right\rangle=F, \\
& g_{2}=\left\langle\alpha_{v v}, N_{2}\right\rangle=\left\langle\alpha_{v}, \alpha_{v}\right\rangle=G,
\end{aligned}
$$

onde $E, F$ e $G$ são os coeficientes da primeira forma fundamental de $\alpha$. Segue que $I_{2}=I$. Agora,

$$
\eta=\frac{I I}{I}=\frac{I I_{1}}{I} N_{1}+\frac{I I_{2}}{I} N_{2}=\frac{I I_{1}}{I} N_{1}+N_{2}
$$

Em outras palavras, nesta situação, a elipse de curvatura $\varepsilon_{\alpha}(p)$ está degenerada em um segmento de reta, sobre a reta suporte $N_{2}=1$, para todo $p \in M$.

Temos

$$
H_{2}(p)=\frac{e_{2} G-2 f_{2} F+E g_{2}}{2\left(E G-F^{2}\right)}=\frac{2\left(E G-F^{2}\right)}{2\left(E G-F^{2}\right)}=1, \forall p \in M .
$$

Disto segue que $H(p) \neq 0$, para todo $p \in M$. Deste modo, se $p$ é uma $H$ singularidade de $M$, então $p$ é necessariamente um ponto de inflexão de $M$. Como sabemos, num ponto de inflexão a elipse de curvatura se torna um segmento de reta radial, ou seja, neste caso a elipse de curvatura se torna um ponto.

A equação diferencial das linhas de curvatura direcional média (2.2.5) tem a forma

$$
0=\operatorname{Jac}\left[\operatorname{Jac}\left(I I_{1}, I_{2}\right), I\right]=\operatorname{Jac}\left[\operatorname{Jac}\left(I_{1}, I\right), I\right],
$$

a qual é a equação diferencial das linhas de curvatura média, considerando $M$ como uma superfície esférica. Garcia e Sotomayor, em [GaS2], estudaram estas linhas sobre superfícies em $R^{3}$. Agora, a equação diferencial das linhas assintóticas (6.2.3) tem a seguinte forma 


$$
0=\operatorname{Jac}\left(I_{1}, I_{2}\right)=\operatorname{Jac}\left(I_{1}, I\right)
$$

a qual nada mais é do que a equação diferencial das linhas de curvatura principal, considerando $M$ como uma superfície esférica. De modo totalmente análogo à seção anterior, vale o seguinte teorema.

Teorema 6.4.1. Suponha que $\alpha: M^{2} \rightarrow S^{3}$ seja uma imersão de uma superfície regular e orientada $M$ em $S^{3}$, com as considerações acima. Então, a equação diferencial quártica (6.2.1) pode ser escrita como o produto

$$
\operatorname{Jac}\left\{J a c\left(I I_{1}, I\right), I\right\} . J a c\left(I I_{1}, I\right)=0 .
$$




\section{Capítulo 7. Estabilidade Estrutural Direcional Média}

Neste trabalho a noção de estabilidade estrutural principal de imersões de superfícies em $R^{3}$, apresentada em [SG1], será adaptada para as linhas de curvatura direcional média das imersões de superfícies em $R^{4}$.

A configuração direcional média de uma imersão $\alpha: M^{2} \rightarrow R^{4}$, onde $M$ é uma superfície regular e orientada, é definida pela terna

$$
\mathscr{R}(\alpha)=\left(S(\alpha), F_{H}(\alpha), f_{H}(\alpha)\right)
$$

onde $S(\alpha)$ é o conjunto das $H$-singularidades, $F_{H}(\alpha)$ é a folheação direcional média máxima e $f_{H}(\alpha)$ é a folheação direcional média mínima de $\alpha$.

A configuração direcional média sintetiza as propriedades qualitativas das folheações $F_{H}(\alpha)$ e $f_{H}(\alpha)$ das imersões de superfícies em $R^{4}$ e representa a maneira que suas linhas se aproximam das $H$-singularidades. Ela é um análogo natural da configuração principal de uma imersão $\alpha: M^{2} \rightarrow R^{3}$ de uma superfície regular e orientada $M$ em $R^{3}$.

Denotemos por $I^{r}=I^{r}\left(M^{2}, R^{4}\right)$ o espaço das imersões de classe $C^{r}$ de $M$ em $R^{4}$ e por $I^{r, s}=I^{r, s}\left(M^{2}, R^{4}\right)$ este espaço munido da topologia $C^{s}$, para $r \geq s$. Uma imersão $\alpha \in I^{r, s}$ é dita ser estruturalmente estável, com relação à 
configuração direcional média, se ela tiver uma vizinhança $V(\alpha)$, tal que, para qualquer $\beta \in V(\alpha)$ existe um homeomorfismo $h: M \rightarrow M$ aplicando $S(\alpha)$ sobre $S(\beta)$, aplicando $F_{H}(\alpha)$ sobre $F_{H}(\beta)$, e aplicando $f_{H}(\alpha)$ sobre $f_{H}(\beta)$.

Agora, uma imersão $\alpha \in I^{r, s}$ é localmente estruturalmente estável em $p \in M$ se para qualquer vizinhança $W_{p}$ de $p$ em $M$, existir uma vizinhança $V(\alpha)$ tal que, para qualquer $\beta \in V(\alpha)$ existem $q=q(\beta) \in W_{p}$, vizinhanças $W_{p}^{1}, W_{q}^{2} \subset W_{p}$ e um homeomorfismo $h: W_{p}^{1} \rightarrow W_{q}^{2}$ aplicando $p$ em $q$, aplicando $F_{H}(\alpha) \cap W_{p}^{1} \quad$ em $\quad F_{H}(\beta) \cap W_{q}^{2}$, bem como aplicando $f_{H}(\alpha) \cap W_{p}^{1} \quad$ em $f_{H}(\beta) \cap W_{q}^{2}$.

De modo análogo podemos definir estabilidade estrutural local em $\gamma$, onde $\gamma \subset M$ é um ciclo de curvatura direcional média.

Teorema 7.1. Seja $p \in M$ uma $H$-singularidade de uma imersão $\alpha \in I^{r, 3}$. Então, $\alpha$ é localmente estruturalmente estável em $p$ se, e somente se, $p$ for uma $H_{\text {- }}$ singularidade de tipo $S$, para todo $r \geq 4$.

Prova. Por uma lado, se $p$ é uma $H$-singularidade de tipo $S$, sua configuração direcional média local é obtida via projeção no plano $u v$ da configuração do campo $X$ em (4.6), conforme teoremas 4.1.2 e 4.2.2. Como neste caso o campo $X$ tem apenas singularidades hiperbólicas ao longo da reta projetiva, a estabilidade estrutural local em $p$ decorre da estabilidade estrutural local das singularidades hiperbólicas. Por outro lado, se $p$ não é uma $H$-singularidade de tipo $S$ pequenas perturbações da imersão $\alpha$ mudam qualitativamente sua configuração local.

De um modo totalmente análogo temos o seguinte teorema. 
Teorema 7.2. Seja $\gamma \subset M$ um ciclo de curvatura direcional média de uma imersão $\alpha \in I^{r, 3}$. Então, $\alpha$ é localmente estruturalmente estável em $\gamma$ se, e somente se, $\gamma$ for um ciclo de curvatura direcional média hiperbólico, para todo $r \geq 4$.

Vejamos a seguir um resultado que será bastante utilizado no próximo capítulo. Denotemos por $I_{c}^{r}=I_{c}^{r}\left(M^{2}, R^{4}\right) \subset I^{r}$ o espaço das imersões de classe $C^{r}$ de $M$ em $R^{4}$, onde $M$ é uma superfície regular, compacta e orientada, e por $I_{c}^{r, s}$ este espaço munido da topologia $C^{s}, \operatorname{com} r \geq s$.

Teorema 7.3. O conjunto das imersões $\alpha \in I_{c}^{r}$ tais que todas as $H$-singularidades são do tipo $S$, é aberto e denso em $I_{c}^{r, 3}$, para todo $r \geq 4$.

Prova. Observemos, primeiramente, que as condições impostas à imersão $\alpha$, próxima de uma $H$-singularidade de tipo $S$, dependem de desigualdades envolvendo derivadas até a terceira ordem. Isto implica a abertura.

Observemos que se a condição de transversalidade for satisfeita em toda $\mathrm{H}$ singularidade, as condições $S_{i}$ podem ser obtidas por um número finito de pequenas mudanças locais nos coeficientes da imersão na carta de Monge, em cada $H$-singularidade. Deste modo, é suficiente considerarmos imersões para as quais a condição de transversalidade se verifica.

O conjunto das $H$-singularidades de uma imersão $\alpha$ é compacto. Portanto, ele pode ser coberto por um número finito de cartas de Monge. A prova do teorema está terminada, bastando para isto aplicar o lema seguinte e a técnica da perturbação passo a passo. 
Lema 7.4. Tomemos a superfície $M$ na carta de Monge, ou seja, $M$ é o gráfico da aplicação $\alpha(u, v)=(u, v, S(u, v), R(u, v))$, com $(u, v) \in U$, sendo este uma vizinhança da origem, que é uma $H$-singularidade, conforme capítulo 4 . Chamemos de $\beta_{a b}$ a imersão

$$
\beta(\alpha ; u, v ; a, b)=\left(u, v, S(u, v)+\frac{a}{2} u^{2}+\frac{b}{2} v^{2}, R(u, v)+a u v+\frac{b}{2} u^{2}\right),
$$

com $(a, b) \in V \subset R^{2}$, onde $V$ é uma vizinhança da origem e $(u, v) \in U$. Então, existe um disco compacto $D \subset U$ e uma vizinhança $V_{a b}=V_{a b}(D) \subset V$, tais que todas as $H$-singularidades da imersão $\beta_{a b}, \operatorname{com}(u, v) \in D$ e $(a, b) \in V_{a b}$, satisfazem a condição de transversalidade. Ou seja, existe um número real positivo $\rho$ tal que a interseção de $V_{a b}(D)$ com o disco de raio $\rho$ tem medida de Lebesgue total.

Prova. Consideremos a equação diferencial das linhas de curvatura direcional média da imersão $\beta_{a b}$, dada por

$$
C(u, v ; a, b) d v^{2}+2 B(u, v ; a, b) d u d v+A(u, v ; a, b) d u^{2}=0,
$$

onde

$$
C=C(u, v ; a, b)=\left[2\left(f_{1} g_{2}-f_{2} g_{1}\right) F+\left(e_{2} g_{1}-e_{1} g_{2}\right) G\right](u, v ; a, b)
$$

$\mathrm{e}$

$$
B=B(u, v ; a, b)=\left[\left(f_{1} g_{2}-f_{2} g_{1}\right) E+\left(e_{2} f_{1}-e_{1} f_{2}\right) G\right](u, v ; a, b) .
$$

No que segue $A(u, v ; a, b)$ não desempenhará papel relevante.

O conjunto

$$
S_{a b}=S\left(\beta_{a b}\right)=\left\{(u, v ; a, b) \in R^{2} \times R^{2} / C(u, v ; a, b)=0=B(u, v ; a, b)\right\}
$$


dos zeros comuns de $C$ e $B$ representa o lugar dos pontos onde a imersão $\beta_{a b}$ tem $H$-singularidades. Nas $H$-singularidades transversais $S_{a b}$ é regular e é, localmente, um gráfico sobre o plano $u v$. De fato, em tais pontos temos

$$
\frac{\partial(C, B)}{\partial(u, v)} \neq 0
$$

No que segue vamos mostrar que se $(a, b)$ está próximo da origem, então $S_{a b}$ é também regular nas $H$-singularidades não transversais. De fato, os níveis $C(u, v ; a, b)=0$ e $B(u, v ; a, b)=0$ interceptam-se transversalmente ao longo da superfície regular $S_{a b}$.

Vamos fazer duas considerações que em nada afetam a generalidade do problema. Se a $H$-singularidade $(0,0)$ for um ponto mínimo fazemos uma rotação no plano normal de modo que os eixos da elipse de curvatura estejam paralelos aos vetores normais $N_{1}$ e $N_{2}$. Se a $H$-singularidade $(0,0)$ for um ponto de inflexão fazemos uma rotação no plano normal de modo que a elipse de curvatura esteja contida na direção $N_{1}$. Com tais considerações e através de extensos cálculos temos

$$
\frac{\partial(C, B)}{\partial(a, b)}(0,0 ; 0,0)=\left(S_{v v}+R_{u u}\right)\left(S_{u u}+S_{v v}+R_{u v}\right)-R_{v v}\left(S_{u v}-R_{u v}\right) \neq 0 .
$$

Deste modo, existem um disco fechado $D \subset U$, suficientemente pequeno, e que contém a origem, e uma vizinhança da origem $V_{a b} \subset V$, suficientemente pequena, tais que se $(u, v) \in D$ e $(a, b) \in V_{a b}$, então $\frac{\partial(C, B)}{\partial(a, b)}(u, v ; a, b) \neq 0$. Isto mostra que $S_{a b}$ é regular. Terminamos a prova do lema utilizando o teorema de Sard e 
identificando o conjunto $V_{a b}$ com os valores regulares da projeção ortogonal de $S_{a b}$ sobre o plano $a b$.

Consideremos o subconjunto $H^{r} \subset I_{c}^{r}$ das imersões $\alpha$ definido pelas seguintes condições:

i) Todas as $H$-singularidades são do tipo $S$ (capítulos 3 e 4);

ii) Todos os ciclos de curvatura direcional média são hiperbólicos (capítulo $5)$;

iii) O conjunto limite de toda linha de curvatura direcional média está contido no conjunto das $H$-singularidades e dos ciclos de curvatura direcional média;

iv) Não existem conexões ou autoconexões de separatrizes das $H$ singularidades (capítulo 4).

Teorema 7.5. O conjunto $H^{r}$ é aberto em $I_{c}^{r, 3}$, e toda $\alpha \in H^{r}$ é estruturalmente estável, para todo $r \geq 4$.

Prova. Considere no fibrado projetivo $P M$ (veja página 35) a superfície $W_{\alpha}$ definida pelas direções de curvatura direcional média. Nas coordenadas $\left(u, v ; p=\frac{d v}{d u}\right)$ esta superfície é definida pela equação diferencial implícita (2.2.2), isto é, $W_{\alpha}=T_{\alpha}^{-1}(0)$, onde $T_{\alpha}(u, v, p)=C(u, v) p^{2}+2 B(u, v) p+A(u, v)$. Esta superfície é regular sob as hipóteses das $H$-singularidades de tipo $S$. A projeção natural $\pi: P M \rightarrow M$ é um duplo recobrimento fora da pré-imagem do conjunto $S(\alpha)$ das $H$-singularidades. 
Em $P M$ está definida a involução $I_{\alpha}(u, v,[d u: d v])=(u, v,[d v:-d u])$ a qual executa uma rotação nas linhas de um ângulo $\pi / 2$. A superfície $W_{\alpha}$ é invariante sob $I$. Sobre $W_{\alpha}$ definimos o campo de direções $X_{\alpha}$ o qual é o levantamento dos campos de direções de curvatura direcional média, que escrito numa carta local $(u, v, p)$ tem a forma

$$
X_{\alpha}(u, v, p)=\left(T_{p}(u, v, p), p T_{p}(u, v, p),-\left(T_{u}(u, v, p)+p T_{v}(u, v, p)\right)\right) .
$$

Este campo tem uma única extensão regular a $\pi^{-1}(S(\alpha))$.

Consideremos o campo de direções induzido $I_{*} X_{\alpha}$, e construamos o par transversal $\left\{X_{\alpha}, I_{*} X_{\alpha}\right\}$ sobre $W_{\alpha} \backslash \pi^{-1}(S(\alpha))$. Este procedimento define uma rede sobre $W_{\alpha}$, fora de $\pi^{-1}(S(\alpha))$. Esta rede tem a propriedade de ser invariante sob $I$ e, além disto, a projeção sob $\pi$ resulta na configuração direcional média.

Com as construções acima podemos estabelecer uma ligação do estudo dos campos de direções de curvatura direcional média com o estudo dos campos de direções principais e suas regiões canônicas, tratadas com detalhes em [GS1]. De fato, aqui a construção e continuação, para uma pequena vizinhança $V(\alpha)$ de $\alpha$, das regiões canônicas segue também da abertura e continuação única, para $\beta$ próximo de $\alpha$, das singularidades (e suas separatrizes e setores parabólicos) e dos ciclos (e suas variedades invariantes locais), devido à hiperbolicidade destes elementos nos campos dos pares $\left\{X_{\alpha}, I_{*} X_{\alpha}\right\}$. Disto podemos concluir a abertura de $H^{r}$ e ainda podemos estabelecer uma correspondência entre $H$-singularidades, separatrizes, ciclos e suas interseções para $\left\{X_{\alpha}, I_{*} X_{\alpha}\right\}$ e $\left\{X_{\beta}, I_{*} X_{\beta}\right\}$. A extensão desta correspondência de modo a estabelecer uma equivalência topológica $\bar{h}: W_{\alpha} \rightarrow W_{\beta}$ entre $\left\{X_{\alpha}, I_{*} X_{\alpha}\right\}$ e $\left\{X_{\beta}, I_{*} X_{\beta}\right\}$, a qual comuta com a involução $I$ e fornece, via projeção, uma equivalência $h: M \rightarrow M$ entre as configurações 
direcionais médias de $\alpha$ e $\beta$ é feita como no caso das redes de direções assintóticas sobre superfícies imersas em $R^{3}$, tratado com detalhes em [GaGS].

Observação 7.6. Pode ser provado que as imersões $\alpha \in I_{c}^{r}$ satisfazendo as condições i), ii) e iv) acima são densas em $I_{c}^{r, 3}$, para todo $r \geq 4$. A condição iii) é mais delicada, uma vez que na demonstração de sua densidade fazemos uso do "Closing Lemma". Como no caso das imersões de superfícies em $R^{3}$, pode ser provado que o conjunto das imersões $\alpha \in I_{c}^{r}$ satisfazendo a condição iii) é denso em $I_{c}^{r, 2}$, para todo $r \geq 4$.

Uma outra questão refere-se aos poucos exemplos que temos em mãos que tratam das imersões de superfícies em $R^{4}$, em particular, exemplos de imersões que pertençam ao conjunto $H^{r}$.

De posse do capítulo 6, podemos responder condicionalmente esta questão. Basta considerar a classe das imersões de superfícies regulares, compactas e orientadas em $R^{3}$ que são estruturalmente estáveis com relação às configurações médias, tratada em detalhes por Garcia e Sotomayor em [GaS2]. Cada uma destas imersões quando composta com a aplicação (inversa da) projeção estereográfica de $R^{3}$ em $S^{3}$ resulta numa imersão de uma superfície regular, compacta e orientada em $R^{4}$ que pertence ao conjunto $H^{r}$. 


\section{Capítulo 8. Topologia e Configuração Direcional Média}

Neste capítulo abordaremos as relações entre a topologia das superfícies e as configurações direcionais médias sobre elas. Faremos também uma análise sobre o conjunto $S(\alpha)$, tecendo alguns comentários a respeito da possibilidade da existência de certos campos de direções tendo singularidades previamente estabelecidas.

A característica de Euler-Poincaré será denotada por $\chi$. Como uma aplicação do teorema 7.3, obtemos os seguintes resultados.

Proposição 8.1. Seja $\alpha: M^{2} \rightarrow R^{4}$ uma imersão de classe $C^{r}, r \geq 4$, de uma superfície regular, compacta, conexa e orientada $M$ em $R^{4}$. Se a curvatura normal de $\alpha$ é positiva (respectivamente negativa) em todo ponto, então

$$
\chi(N M)=2 \chi(M)(\text { respectivamente } \chi(N M)=-2 \chi(M))
$$

Prova. É suficiente provarmos o teorema para um subconjunto denso $T^{r} \subset \bar{I}_{c}^{r}$, onde $\bar{I}_{c}^{r}=I_{c}^{r} \cap I_{k_{N}}^{r}$ e $I_{k_{N}}^{r}$ é o conjunto das imersões de classe $C^{r}$ de $M$ em $R^{4}$, tais 
que a curvatura normal é positiva (ou negativa) em todo ponto. De fato, se $\beta \in \bar{I}_{c}^{r}$ então existe uma seqüência $\left(\alpha_{n}\right)_{n \in N}$ em $T^{r}$, tal que $\alpha_{n} \rightarrow \beta$.

Agora, para imersões de superfícies compactas e orientadas temos ([Lit], página 291)

$$
\chi(N M)=\frac{1}{2 \pi} \int_{M} k_{N} d A .
$$

Se o teorema é válido para $T^{r}$, então

$$
\pm 2 \chi(M)=\chi\left((N M)_{\alpha_{n}}\right)=\frac{1}{2 \pi} \int_{M} k_{N}\left(\alpha_{n}\right) d A \rightarrow \frac{1}{2 \pi} \int_{M} k_{N}(\beta) d A=\chi(N M)_{\beta}
$$

Tomemos o subconjunto $T^{r} \subset \bar{I}_{c}^{r}$ das imersões tais que todas as $H$ singularidades são do tipo $S$. Segue, via teorema 7.3 que $T^{r} \subset \bar{I}_{c}^{r}$ é denso, para todo $r \geq 4$. Ademais, toda $\alpha \in T^{r}$ tem um número finito, digamos $p_{1}, \ldots, p_{n}$, de $H$-singularidades, uma vez que $M$ é compacta e as $H$-singularidades de tipo $S$ são isoladas. Como $k_{N}(p) \neq 0, \forall p \in M$, cada ponto $p_{j}, j=1, \ldots, n$, é uma singularidade do campo de vetores curvatura média, isto é,

$$
H\left(p_{j}\right)=0, j=1, \ldots, n \text {. }
$$

Do teorema 3.1.3, temos

$$
\chi(N M)=\sum_{j=1}^{n} I\left(H, p_{j}\right)=\sum_{j=1}^{n} \pm 2 i\left(p_{j}\right)= \pm 2 \sum_{j=1}^{n} i\left(p_{j}\right)= \pm 2 \chi(M) .
$$

O sinal \pm concorda com o sinal da curvatura normal. 
Corolário 8.2. Seja $\alpha: M^{2} \rightarrow R^{4}$ uma imersão de classe $C^{r}, r \geq 4$, de uma superfície regular, compacta, conexa e orientada $M$ em $R^{4}$. Se $M$ não é homeomorfa a $S^{2}$ então existe um ponto $p \in M$, tal que $k_{N}(p)=0$.

Prova. Suponhamos que $k_{N}(q) \neq 0, \forall q \in M$. Então a curvatura normal de $\alpha$ é positiva (o caso negativo é análogo) em todo ponto. Pela proposição 8.1, temos

$$
\chi(M)=\frac{1}{2} \chi(N M)=\frac{1}{4 \pi} \int_{M} k_{N} d A>0
$$

Segue que $M$ é homeomorfa a $S^{2}$, e isto prova o corolário.

Os resultados acima foram provados por Little, em [Lit], e por Asperti, em [Asp], utilizando uma outra caracterização para o conjunto $T^{r}$.

Proposição 8.3. Genericamente uma esfera de dimensão dois $C^{r}$ mergulhada, $r \geq 4$, como uma superfície localmente convexa em $R^{4}$ tem, no mínimo, quatro pontos de inflexão.

Prova. Genericamente, temos

$$
\# S(\alpha) \geq 2|\chi(M)|=4 \text {. }
$$

Mas,

$$
\# S(\alpha)=\# S_{1}(\alpha)+\# S_{2}(\alpha)
$$

onde $S_{1}(\alpha)$ e $S_{2}(\alpha)$ estão definidos na observação 2.2.3. Sobre uma superfície localmente convexa a origem de $N_{p} M$ não pertence à região limitada pela elipse de curvatura, para todo $p \in M$, conforme [GMFR]. Portanto,

$$
S_{1}(\alpha)=\varnothing
$$


Segue que

$$
\# S_{2}(\alpha) \geq 4
$$

A proposição 8.3 foi provada em [GMFR] utilizando os pontos de inflexão como singularidades dos campos de direções assintóticas.

Façamos agora alguns comentários a respeito do conjunto $S(\alpha)$ das $H$ singularidades de uma imersão $\alpha: M^{2} \rightarrow R^{4}$. Primeiro, pensemos na possibilidade de construirmos um determinado campo de direções tendo como conjunto de suas singularidades o conjunto $S_{1}(\alpha)$ dos pontos mínimos de $\alpha$. Tomemos agora uma imersão de uma superfície compacta, de característica de Euler-Poincaré não nula, em $R^{3}$. Façamos a composição desta imersão com a projeção estereográfica de $R^{3} \mathrm{em} S^{3}$, obtendo assim uma imersão de uma superfície compacta, de característica de Euler-Poincaré não nula, em $R^{4}$. Tomemos o suposto campo de direções sobre esta imersão. Como a característica de Euler-Poincaré é diferente de zero, a soma dos índices das singularidades isoladas deste campo de direções, os pontos mínimos, deve ser diferente de zero. Mas isto nos leva a uma contradição, pois, como vimos no capítulo 6, o vetor curvatura média é diferente de zero em todo ponto, isto é, o conjunto dos pontos mínimos é vazio.

Em outras palavras, não é possível a construção de um campo de direções sobre uma superfície em $R^{4}$ possuindo singularidades apenas nos pontos mínimos da superfície. 


\section{Capítulo 9. Conclusões}

Passemos agora a abordar alguns pontos em aberto que apareceram ao longo deste trabalho e que constituem interessante material para estudo futuro.

$\mathrm{O}$ primeiro assunto refere-se à obtenção de um campo de direções sobre uma superfície imersa em $R^{4}$ com a seguinte propriedade: quando, em particular, a imersão estiver contida em um hiperplano, as curvas integrais deste campo devem coincidir com as linhas assintóticas das imersões de superfícies em $R^{3}$. Tal campo mereceria a denominação de campo de direções assintóticas.

Um segundo assunto refere-se ao estudo de uma equação diferencial quártica da forma

$$
a_{4}(u, v) d v^{4}+a_{3}(u, v) d v^{3} d u+a_{2}(u, v) d v^{2} d u^{2}+a_{1}(u, v) d v d u^{3}+a_{0}(u, v) d u^{4}=0,
$$

nos moldes do trabalho [BT]. Uma aplicação imediata deste estudo seria a equação diferencial quártica das linhas de curvatura axial (6.2.1).

Um terceiro assunto, mais amplo, refere-se à seguinte situação: para cada ponto da imersão $\alpha: M^{2} \rightarrow R^{4}$, escolha na elipse de curvatura dois pontos distintos simétricos com relação ao centro da elipse de curvatura, segundo um critério previamente estabelecido. Induza no espaço tangente o par de direções ortogonais determinado por estes pontos. Faça isto para todos os pontos da 
imersão. Temos assim uma receita para a construção de um campo de direções. Admitamos a possibilidade de escrevermos a equação diferencial deste campo. Tal equação diferencial deve ser necessariamente quadrática. Uma questão pode ser assim colocada. Serão as singularidades genéricas deste campo análogas às $H$ singularidades de tipo $S$ ? Em outras palavras, será a equação diferencial deste campo uma equação diferencial binária da forma

$$
A(u, v) d u^{2}+B(u, v) d u d v-A(u, v) d v^{2}=0 ?
$$

Ou pode ocorrer a forma

$$
A(u, v) d u^{2}+B(u, v) d u d v+A(u, v) d v^{2}=0 ?
$$

Aqui $(u, v)$ é uma carta positiva de $M$. 


\section{Referências Bibliográficas}

[Asp] A. C. Asperti, Immersions of surfaces into 4-dimensional spaces with nonzero normal curvature, Ann. Mat. Pura Appl., 125 (1980), 313-329.

[BT] J. W. Bruce e F. Tari, On binary differential equations, Nonlinearity, 8 (1995), 255-271.

[Car] M. P. do Carmo, Differential geometry of curves and surfaces, PrenticeHall, New Jersey, (1976).

[Dar] G. Darboux, Sur la forme des lignes de courbure dans la voisinage d'un ombilic, Note 07, Leçons sur la théorie de surfaces, vol. IV, Gauthier Villars, Paris, (1896).

[Eul] L. Euler, Recherches sur la courbure des surfaces, Mémoires de 1'Academie de Sciences de Berlin, 16, (1760).

[For] A. R. Forsyth, Geometry of four dimensions, vols. I e II, Cambridge Univ. Press, Cambridge, (1930).

[Ga1] R. A. Garcia, Linhas de curvatura de hipersuperficies imersas no espaço $R^{4}$, Tese de doutoramento, IMPA, (1989).

[GaS1] R. Garcia e J. Sotomayor, Lines of axial curvature on surfaces immersed in $R^{4}$, Diff. Geom. And its Applications, 12 (2000), 253-269. 
[GaS2] R. Garcia e J. Sotomayor, Structurally stable configurations of lines of mean curvature and umbilic points on surfaces immersed in $R^{3}$, a ser publicado em Publicacions Matemàtiques, volume 45, número 2, (2001).

[GaGS] R. Garcia, C. Gutierrez e J. Sotomayor, Structural stability of asymptotic lines on surfaces immersed in $R^{3}$, Bulletin de Sciences Mathematiques, 123 (1999), 599-622.

[GGST] C. Gutierrez, I. Guadalupe, J. Sotomayor e R. Tribuzy, Principal lines on surfaces minimally immersed in constantly curved 4-spaces, Dynamical Systems and Bifurcation Theory, 160 (1987), 91-120.

[GGTG] C. Gutierrez, I. Guadalupe, R. Tribuzy e V. Guíñez, Lines of curvature on surfaces immersed in $R^{4}$, Bol. Soc. Bras. Mat., 28 (1997), 233-251.

[GMFR] R. Garcia, D. Mochida, M. R. Fuster e M. S. Ruas, Inflection points and topology of surfaces in 4-space, Trans. Am. Math. Soc., 352 (2000), 3029-3043. [GS1] C. Gutierrez e J. Sotomayor, Lines of curvature and umbilical points on surfaces, $18^{\circ}$ Colóquio Brasileiro de Matemática, IMPA, Rio de Janeiro, (1991). [GS2] C. Gutierrez e J. Sotomayor, Lines of curvature, umbilic points and Carathéodory Conjecture, Resenhas do IME-USP, 3 (1998), 291-322.

[Hop] H. Hopf, Differential geometry in the large, Lectures Notes in Math. 1000, Springer-Verlag, (1989).

[Lit] J. A. Little, On singularities of submanifolds of a higher dimensional Euclidean space, Ann. Mat. Pura Appl., 83 (1969), 261-335.

[Mon] G. Monge, Sur les lignes de courbure de la surface de l'ellipsoide, Journ. de l'Ecole Polytech., II cah., (1796).

[Pei] M. Peixoto, Structurally stable vector fields on two-dimensional manifolds, Topology, 1 (1962), 101-120. 
[SMe] J. Sotomayor e L. F. Mello, A note on some developments on Carathéodory conjecture on umbilic points, Expositiones Mathematicae, 17 (1999), 49-58.

[Sot] J. Sotomayor, O elipsóide de Monge, Matemática Universitária, 15 (1993), 33-47.

[Won] W. C. Wong, A new curvature theory for surfaces in euclidean 4-spaces, Comm. Math. Helv., 26 (1952), 152-170. 